This is the final peer-reviewed accepted manuscript of:

Zattoni, Elena, Anna Maria Perdon, and Giuseppe Conte. 2018. "Measurement Dynamic Feedback Output Regulation in Hybrid Linear Systems with State Jumps." International Journal of Robust \& Nonlinear Control 28 (2): 416-36.

The final published version is available online at:

https://doi.org/10.1002/rnc.3875

Rights / License:

The terms and conditions for the reuse of this version of the manuscript are specified in the publishing policy. For all terms of use and more information see the publisher's website.

This item was downloaded from IRIS Università di Bologna (https://cris.unibo.it/)

When citing, please refer to the published version. 


\title{
Measurement dynamic feedback output regulation in hybrid linear systems with state jumps
}

\author{
E. Zattoni ${ }^{1 *}$, A.M. Perdon ${ }^{2}$ and G. Conte ${ }^{2}$ \\ ${ }^{1}$ Department of Electrical, Electronic, and Information Engineering "G. Marconi" \\ Alma Mater Studiorum - University of Bologna, 40136 Bologna, Italy \\ ${ }^{2}$ Department of Information Engineering, Polytechnic University of Marche, 60131 Ancona, Italy
}

\begin{abstract}
SUMMARY
This work investigates the problem of designing a hybrid dynamic feedback regulator that forces the output of a hybrid linear system to asymptotically converge to the reference generated by a hybrid exogenous system, asymptotically rejects the exogenous disturbance, and attains global asymptotic stability of the compensated hybrid linear dynamics. The class of hybrid linear systems addressed exhibits a continuoustime linear behavior except at isolated points of the time axis, where the state is subject to discontinuities caused by a jump behavior. In the presence of possibly unequally spaced state jumps, under the only constraint that the minimum time between any two consecutive jumps is no smaller than a given positive real constant, both implicit and explicit sufficient conditions for the existence of a solution to the stated problem are shown. The explicit condition is constructive, in the sense that it outlines the algorithmic procedure for the synthesis of the hybrid feedback regulator, provided that a certain output-nulling hybrid controlled invariant subspace be known. Then, a necessary and sufficient condition for the existence of such subspace is proven, so that a computational means to derive it, if any exists, is given. Finally, the devised approach is applied to a numerical example borrowed from the literature, with the twofold aim of illustrating its implementation and making a comparison with the available method.
\end{abstract}

Received April 14, 2017

KEY WORDS: hybrid linear systems; linear impulsive systems; output regulation; dynamic feedback; global asymptotic stability; dwell time.

*Correspondence to: Elena Zattoni, Department of Electrical, Electronic, and Information Engineering "G. Marconi”, Alma Mater Studiorum - University of Bologna, Viale del Risorgimento 2, 40136 Bologna, Italy. E-mail: elena.zattoni@unibo.it 


\section{INTRODUCTION}

Hybrid linear systems with state jumps (or linear impulsive systems) are dynamical systems featuring continuous-time state motions (flow dynamics) interrupted by state discontinuities occurring at isolated time instants (jump dynamics). This class of hybrid dynamical systems has attracted noticeable interest in the last decade, owing to its effectiveness in modeling the special way some real systems, present in several fields of engineering and science, behave [1-3]. Classic control problems recently extended to linear impulsive systems are stabilization by state or output feedback [4-6], state estimation [7], linear quadratic control [8,9], disturbance decoupling [10-12], and output regulation [13-21]. Less typical formulations of some of these problems have also been investigated for hybrid linear systems: see, e.g., [22] on almost output regulation and [23, 24] on finite-time stabilization and control.

In this context, the aim of this paper is to contribute a deeper insight and new methodological tools to solve a quite general formulation of the output regulation problem. The discussion is focused on hybrid linear systems with time-driven, possibly non-uniformly spaced, not a-priori known state jumps, meeting the only constraint that the length of the time interval between any two subsequent jumps is greater than or equal to a given positive real constant (dwell time). The problem addressed consists in finding a hybrid linear regulator, subject to state jumps synchronous with those affecting the plant and the exogenous system, such that the plant regulated output asymptotically converges to the reference generated by the exogenous system, the exogenous disturbance is asymptotically rejected, and the dynamics of the hybrid linear system compensated by measurement dynamic feedback is globally asymptotically stable for all the admissible jump time sequences. First, a sufficient condition for problem solvability is given in terms of structural and stability properties of a hybrid invariant subspace of the overall autonomous hybrid system. Then, a sufficient condition for problem solvability is expressed in terms of structural and stabilizability properties of an outputnulling hybrid controlled invariant subspace of the connection between the plant and the exogenous system (extended hybrid system). The first condition is interesting for two main reasons. On the one hand, it provides insight on how the overall system's motion should evolve so as to guarantee that all the design targets are achieved. On the other hand, it is functional to the proof of the second sufficient condition. The latter one, compared to the former, has the advantage of being constructive: i.e., the proof shows how to synthesize the hybrid feedback regulator, provided that the mentioned output-nulling hybrid controlled invariant subspace be known. Further, a necessary and sufficient condition for the existence of such output-nulling hybrid controlled invariant subspace is proven. More precisely, the necessary and sufficient condition gives the computational tools to ascertain the existence of that subspace and, if it exists, it shows how to determine it. Concerning the methodological aspect, this work extensively uses the geometric approach, that has recently proven 
to be very effectual in solving control problems for other classes of hybrid systems, like switching linear systems [25-28]. This work differs from the cited papers [13-21] on output regulation in several aspects, as is detailed below.

As to [13], a main difference concerns the nature of the input signals and of the measured outputs. In [13], the signal to be tracked or rejected is a discrete sequence, defined at the jump times and applied to the jump dynamics of the hybrid plant. In this work, the reference signal and the exogenous disturbance are piecewise-continuous functions generated by the hybrid exogenous system: the former is compared to the piecewise-continuous regulated output of the hybrid plant, while the latter affects both the plant dynamics. Likewise, in [13] the measured output is a discrete sequence, while in this work it is a piecewise-continuous function. The structure of the feedback regulator differs consequently: in [13] the feedback variable is applied to the regulator jump dynamics, while in this work it is applied to the regulator flow dynamics. Furthermore, the fact that in [13] the signal to be tracked or rejected is not generated by an exogenous system implies that the solution presented therein is more similar to that typical of disturbance decoupling, where no information is available on the disturbance, rather than to that of output regulation as described, for instance, in the basic reference [29] or in the geometric revisiting [30]. Nonetheless, it must be acknowledged that [13] represents the first attempt to extend the geometric control theory to linear impulsive systems (though to a special class of them, with the characteristics specified above) and that some ideas presented therein have fostered further interesting developments [31,32].

Concerning [14-17], the primary distinction lies in the hybrid time domain. In all those papers, the admissible jump time sequences are periodic. This discrepancy in the problem formulation impacts on stability in such a way that the comparison between the solvability conditions appears meaningless. Nonetheless, it is worth pointing out other differences in the problem statement, specifically concerning the structure of the plant and of the compensator, since some of them also remain in those papers by the same authors where the assumption of periodic jumps is removed [20, 21]. Indeed, in this work, the plant has two output variables (the regulated output and the measured output), while in all the abovementioned papers only one variable (the performance output) plays both roles. Moreover, in this work, the plant is subject to a control input applied to the flow dynamics and affecting the regulated output through a feedthrough term, while the jump dynamics is free. None of the abovementioned papers considers this layout. In fact, [14,16], which are the only ones, among those mentioned, to consider a free jump dynamics, do not include any feedthrough term. The various definitions of the plant obviously influence the structure of the regulator. The companion papers $[14,15]$ discuss a compensation scheme with a twofold structure, compared to the compact one considered herein, since it consists of a trajectory generator and a stabilizer. Instead, both [16] and [17] consider two different compensators: i.e., a static full information regulator and an error feedback regulator. With respect to these latter, the hybrid linear regulator proposed herein, which 
is aimed at asymptotically annhilating the regulation error by performing a measurement dynamic feedback, has not only the advantage of dropping the assumption of full information on the plant state (as can be achieved by error dynamic feedback), but it also has the advantage of taking into account that the measured variable may not coincide with the regulation error. In addition to the relevant dissimilarities described above, it is worth mentioning the different methodologies adopted, although a deeper discussion of this aspect seems more appropriate when comparing this work with $[20,21]$, which deal with a more similar problem, at least as far as the definition of the hybrid time domain is concerned.

Hybrid linear systems with periodic jumps are also addressed in [19] (as well as in the earlier conference paper [18]). Since this work shares with [19] the geometric approach, the impact of dropping the assumption of periodic jumps is evident from their comparison. In particular, concerning the implicit solvability condition (i.e., the condition pertaining to the overall compensated system), necessity does not hold anymore. The main reason is that the notion of state transition map of the hybrid linear system over one period is lost. This fact has a twofold consequence: invariance can only be separately referred to the linear maps that respectively define the flow and the jump dynamics, meanwhile stability cannot be associated to one suitably-defined linear map anymore. On this basis, only more demanding requirements for solvability of the problem with stability can be formulated, which, in turn, are only sufficient. As to the explicit solvability condition (namely, the condition referred to the extended hybrid system), a more general definition of the plant calls for enhanced geometric tools compared to those employed in [19]. More precisely, the distinction between the regulated output and the measured output, along with the presence of a feedthrough term from the control input to the regulated output, require that the notion of output-nulling hybrid controlled invariant subspace be introduced, which brings in new properties and algorithms, needed to achieve the problem solution.

The papers $[20,21]$ are more strictly related to this one as to the class of hybrid linear systems addressed, which are hybrid linear systems with possibly non-uniformly spaced, not a-priori known state jumps. In both papers, the compensator devised consists in a full information, static hybrid regulator - i.e., the control inputs on the flow and jump dynamics of the plant are given by static feedbacks of the states of the plant and of the exogenous system. Indeed, complete knowledge of the plant and of the exosystem states may be a rather retrictive assumption. Instead, as previously remarked, the hybrid linear regulator considered herein performs a measurement dynamic feedback and, as such, does not require state knowledge. In addition to this basic distinction, there exist some other relevant differences between the methodological approach developed in this work and those presented in [20,21]. Indeed, both [20] and [21] encompass a geometric perspective on output regulation for hybrid linear systems. In particular, [21] relates solvability of the algebraic output regulator equations to the existence of the maximal hybrid controlled invariant subspace 
contained in the null space of the output for the extended hybrid system. Instead, [20] leaves the regulator equations apart from consideration and sets forth geometric arguments centered on the abovementioned subspace. In this regard, it is worth noting that the presence of the feedthrough term from the control input to the regulated output considered in this work imposes to refer to a technically different notion of hybrid controlled invariance - i.e., output-nulling hybrid controlled invariance. Nevertheless, a comparison carried out despite this discrepancy shows an even more important differentiation in the way of dealing with stability. In fact, both [20] and [21] are first focused on the computation of the maximal hybrid controlled invariant subspace and then on the analysis of the possibility of stabilizing their inner and outer hybrid dynamics. Instead, the explicit sufficient condition presented in this work is focused on an output-nulling hybrid controlled invariant subspace which satisfies the minimal structural requisite, while its inner and outer stabilizability follow from the hypotheses on the plant and on the extended hybrid system.

Lastly, it is worth considering [22]. Indeed, the problem dealt with in [22] is an almost output regulation problem, since, in addition to output regulation, the minimization of a weighted $\mathscr{L}_{2}$-norm from a pertubation input to the error is required. Moreover, global asymptotic stability of the closedloop hybrid dynamics is achieved under an average dwell time constraint, on the assumptions of stabilizability of the plant flow dynamics and of detectability of the extended system flow dynamics. Nonetheless, the problem tackled in [22] reduces to exact output regulation when the $\mathscr{L}_{2}$-requirement is dropped. Hence, the comparison between this work and [22] makes sense only in this special case and points out a substantially different methodological approach. Both papers express sufficient-only conditions for problem solvability. However, in [22] they are expressed in terms of solvability of a set of linear matrix equations and linear matrix inequalities, while in this work they are given in terms of structural and qualitative properties of suitably-defined geometric subspaces. In particular, as far as stability is concerned, a Lyapunov-like function is adopted in [22], while this work refers to stability of the hybrid linear dynamics associated to the considered geometric subspaces. As to the dwell time, while in [22] global asymptotic stability is achieved for an average dwell time, in this work it is attained for any fixed and given dwell time. In order to better illustrate the characteristics of the approach discussed in this work with respect to [22], such approach has been successfully applied to the numerical example devised in [22].

This work is organized as follows. Section 2 introduces the output regulation problem with global asymptotic stability of the compensated hybrid dynamics. Section 3 provides a sufficient condition for the existence of a solution to the stated problem, expressed in terms of structural and stability properties of a hybrid invariant subspace of the overall autonomous hybrid system (implicit condition). Section 4 shows a sufficient condition for problem solvability, based on structural and stabilizability properties of an output-nulling hybrid controlled invariant subspace of the extended hybrid system (explicit condition). Section 5 presents a necessary and sufficient condition for the 
existence of the output-nulling hybrid controlled invariant subspace playing the key role in the explicit condition. Section 6 illustrates the application of the proposed approach to a numerical example taken from the literature. Section 7 reports some concluding remarks. Appendix describes a technique for globally asymptotically stabilizing a hybrid linear dynamics by applying a static state feedback to the flow dynamics.

Notation: The symbols $\mathbb{R}$ and $\mathbb{R}^{+}$stand for the sets of real numbers and nonnegative real numbers, respectively. Matrices and linear maps are denoted by slanted capital letters, like $A$. Sets, vector spaces, and subspaces are denoted by calligraphic letters, like $\mathcal{X}$. The notation $\mathcal{P} \oplus \mathcal{V}=\mathcal{X}$, where $\mathcal{P}$ and $\mathcal{V}$ are subspaces of a vector space $\mathcal{X}$, stands for $\mathcal{P}+\mathcal{V}=\mathcal{X}$ and $\mathcal{P} \cap \mathcal{V}=\{0\}$. When the condition $\mathcal{P} \oplus \mathcal{V}=\mathcal{X}$ holds, $\mathcal{V}$ is said to be a direct summand of $\mathcal{P}$ (and viceversa). The quotient space of a vector space $\mathcal{X}$ over a subspace $\mathcal{V} \subseteq \mathcal{X}$ is denoted by $\mathcal{X} / \mathcal{V}$. The restriction of a linear map $A$ to an $A$-invariant subspace $\mathcal{V}$ is denoted by $\left.A\right|_{\mathcal{V}}$. The map induced by $A$ on the quotient space $\mathcal{X} / \mathcal{V}$ is denoted by $\left.A\right|_{\mathcal{X} / \mathcal{V}}$. The image and the kernel of $A$ are denoted by $\operatorname{Im} A$ and $\operatorname{Ker} A$, respectively. The notation $T^{-1}$ stands for the inverse of the nonsingular square matrix $T$. The notation $A^{-1} \mathcal{V}$, where $A$ is a not necessarily invertible linear map and $\mathcal{V}$ is a subspace, stands for the inverse image of $\mathcal{V}$ through $A$. The notation $\max \mathcal{J}(A, \mathcal{K})$ stands for the maximal $A$-invariant subspace contained in the subspace $\mathcal{K}$. The symbols $0_{m \times n}$ and $I_{n}$ respectively stand for an $m \times n$ zero matrix and an identity matrix of dimension $n$ (subscripts are dropped when the dimensions can be inferred from the context). The symbol diag $\left\{d_{1}, \ldots, d_{n}\right\}$ stands for the $n \times n$ diagonal matrix whose nonzero entries are $d_{1}, \ldots, d_{n}$, respectively. The symbol $\|x\|$, where $x \in \mathbb{R}^{n}$, denotes the Euclidean norm of $x$ and the symbol $\|M\|$, where $M \in \mathbb{R}^{m \times n}$, denotes the matrix 2-norm, defined by $\|M\|=\sup _{x \in \mathbb{R}^{n}, x \neq 0}\|M x\| /\|x\|$.

\section{PROBLEM STATEMENT}

The aim of this section is to introduce the problem of output regulation by measurement dynamic feedback, with global asymptotic stability of the compensated dynamics, for hybrid linear systems featuring possibly non-uniformly spaced, not a-priori known state jumps, under the only constraint of a minimum dwell time between any two consecutive jumps.

The hybrid time domain is defined through the following notation. A finite or countably infinite ordered set $\left\{t_{0}, t_{1}, \ldots\right\}$ of strictly increasing elements of $\mathbb{R}^{+}$, with no accumulation points, is denoted by $\mathcal{T}$ - when $\mathcal{T}$ is finite, $t_{f}$ stands for the last element. The nonnegative real axis except the elements of $\mathcal{T}$ is denoted by $\mathbb{R}^{+} \backslash \mathcal{T}$. The minimum difference between two consecutive elements of $\mathcal{T}$ (also including the difference between the first element of $\mathcal{T}$ and the origin of $\mathbb{R}^{+}$) is denoted by $\tau_{\min }$. Given a positive real constant $\tau$, the set of all $\mathcal{T}$ such that $\tau_{\min } \geq \tau$ is denoted by $\mathscr{T}_{\tau}$. It is worth noting that the assumption that the set $\mathcal{T}$ has no accumulation points is required in order to 
guarantee that the hybrid linear systems that hinge on the hybrid time domain thus defined do not exhibit Zeno behaviors.

The plant is the hybrid linear system

$$
\Sigma_{P} \equiv\left\{\begin{aligned}
\dot{x}_{P}(t) & =A_{P} x_{P}(t)+B_{P} u(t)+H_{P} x_{E}(t), \quad t \in \mathbb{R}^{+} \backslash \mathcal{T}, \\
x_{P}\left(t_{k}\right) & =J_{P} x_{P}^{-}\left(t_{k}\right)+K_{P} x_{E}^{-}\left(t_{k}\right), \quad t_{k} \in \mathcal{T}, \\
y_{R}(t) & =E_{P} x_{P}(t)+D_{P} u(t), \quad t \in \mathbb{R}^{+}, \\
y_{M}(t) & =C_{P} x_{P}(t), \quad t \in \mathbb{R}^{+},
\end{aligned}\right.
$$

where $x_{P} \in \mathcal{X}_{P}=\mathbb{R}^{n_{P}}$ is the state, $u \in \mathcal{U}=\mathbb{R}^{p}$ is the control input, $x_{E} \in \mathcal{X}_{E}=\mathbb{R}^{n_{E}}$ is the exogenous signal, $y_{R} \in \mathcal{Y}_{R}=\mathbb{R}^{q}$ is the regulated output, and $y_{M} \in \mathcal{Y}_{M}=\mathbb{R}^{m}$ is the measured output, with $p, q, m \leq n_{P} . A_{P}, B_{P}, H_{P}, J_{P}, K_{P}, E_{P}, D_{P}$, and $C_{P}$ are constant real matrices of suitable dimensions. The matrices

$$
\left[\begin{array}{c}
B_{P} \\
D_{P}
\end{array}\right], \quad\left[\begin{array}{ll}
E_{P} & D_{P}
\end{array}\right],
$$

and $C_{P}$ are assumed to be full-rank. The set of the admissible input functions $u(t)$, with $t \in \mathbb{R}^{+}$, is assumed to be the set of all piecewise-continuous functions with values in $\mathbb{R}^{p}$.

The exogenous system is the hybrid linear system

$$
\Sigma_{E} \equiv\left\{\begin{aligned}
\dot{x}_{E}(t) & =A_{E} x_{E}(t), & & t \in \mathbb{R}^{+} \backslash \mathcal{T}, \\
x_{E}\left(t_{k}\right) & =J_{E} x_{E}^{-}\left(t_{k}\right), & & t_{k} \in \mathcal{T}, \\
d_{R}(t) & =E_{E} x_{E}(t), & & t \in \mathbb{R}^{+}, \\
d_{M}(t) & =C_{E} x_{E}(t), & & t \in \mathbb{R}^{+},
\end{aligned}\right.
$$

where $x_{E} \in \mathbb{R}^{n_{E}}$ is the state, $d_{R} \in \mathbb{R}^{q}$ is the reference for the regulated output, and $d_{M} \in \mathbb{R}^{m}$ is the reference for the measured output. $A_{E}, J_{E}, E_{E}$, and $C_{E}$ are constant real matrices. $E_{E}$ and $C_{E}$ are assumed to be full-rank. It is worth noting that the equations of the plant $\Sigma_{P}$ presuppose that the state of the exogenous system $\Sigma_{E}$ is directly accessible.

The differential state equations of $\Sigma_{P}$ and $\Sigma_{E}$ rule the so-called flow dynamics. The algebraic state equations rule the jump dynamics. Hence, the state evolutions $x_{P}(t)$ and $x_{E}(t)$ in the time interval $\left[0, t_{0}\right)$ are the solution of the system of differential equations, given the initial conditions $x_{P}(0)=x_{P 0}$ and $x_{E}(0)=x_{E 0}$, along with the input function $u(t)$, with $t \in\left[0, t_{0}\right)$. The states $x_{P}\left(t_{k}\right)$ and $x_{E}\left(t_{k}\right)$, with $t_{k} \in \mathcal{T}$, are obtained by the images through $J_{P}, K_{P}$, and $J_{E}$ of $x_{P}^{-}\left(t_{k}\right)=\lim _{\varepsilon \rightarrow 0^{+}} x_{P}\left(t_{k}-\varepsilon\right)$ and $x_{E}^{-}\left(t_{k}\right)=\lim _{\varepsilon \rightarrow 0^{+}} x_{E}\left(t_{k}-\varepsilon\right)$. The state evolutions $x_{P}(t)$ and $x_{E}(t)$ in $\left[t_{k}, t_{k+1}\right)$, with $t_{k}, t_{k+1} \in \mathcal{T}$, are the solutions of the system of differential equations, given the initial conditions $x_{P}\left(t_{k}\right)$ and $x_{E}\left(t_{k}\right)$, along with the input function $u(t)$, with $t \in\left[t_{k}, t_{k+1}\right)$.

The regulation error $e_{R} \in \mathbb{R}^{q}$ and the measured error $e_{M} \in \mathbb{R}^{m}$ are respectively defined by

$$
\begin{gathered}
e_{R}(t)=d_{R}(t)-y_{R}(t), \quad t \in \mathbb{R}^{+}, \\
e_{M}(t)=d_{M}(t)-y_{M}(t), \quad t \in \mathbb{R}^{+} .
\end{gathered}
$$


The feedback regulator is the hybrid linear system

$$
\Sigma_{R} \equiv\left\{\begin{aligned}
\dot{x}_{R}(t) & =A_{R} x_{R}(t)+B_{R} e_{M}(t), \quad t \in \mathbb{R}^{+} \backslash \mathcal{T}, \\
x_{R}\left(t_{k}\right) & =J_{R} x_{R}^{-}\left(t_{k}\right), \quad t_{k} \in \mathcal{T}, \\
u(t) & =C_{R} x_{R}(t)+D_{R} e_{M}(t), \quad t \in \mathbb{R}^{+},
\end{aligned}\right.
$$

where $x_{R} \in \mathbb{R}^{n_{R}}$ is the state and $e_{M} \in \mathbb{R}^{m}$ is the measured error. $A_{R}, B_{R}, J_{R}, C_{R}$, and $D_{R}$ are to-be-designed constant real matrices.

In order to state the output regulation problem, the hybrid linear system $\Sigma_{L}$ is defined as the closed-loop connection of the plant and the feedback regulator, so that

$$
\Sigma_{L} \equiv\left\{\begin{aligned}
\dot{x}_{L}(t) & =A_{L} x_{L}(t)+B_{L} x_{E}(t), \quad t \in \mathbb{R}^{+} \backslash \mathcal{T}, \\
x_{L}\left(t_{k}\right) & =J_{L} x_{L}^{-}\left(t_{k}\right)+K_{L} x_{E}^{-}\left(t_{k}\right), \quad t_{k} \in \mathcal{T}, \\
e_{R}(t) & =E_{L} x_{L}(t)+D_{L} x_{E}(t), \quad t \in \mathbb{R}^{+},
\end{aligned}\right.
$$

where

$$
\begin{aligned}
& A_{L}=\left[\begin{array}{cc}
A_{P}-B_{P} D_{R} C_{P} & B_{P} C_{R} \\
-B_{R} C_{P} & A_{R}
\end{array}\right], \quad B_{L}=\left[\begin{array}{c}
B_{P} D_{R} C_{E}+H_{P} \\
B_{R} C_{E}
\end{array}\right], \\
& J_{L}=\left[\begin{array}{cc}
J_{P} & 0 \\
0 & J_{R}
\end{array}\right], \quad K_{L}=\left[\begin{array}{c}
K_{P} \\
0
\end{array}\right], \\
& E_{L}=\left[\begin{array}{cc}
-\left(E_{P}-D_{P} D_{R} C_{P}\right) & -D_{P} C_{R}
\end{array}\right], \quad D_{L}=E_{E}-D_{P} D_{R} C_{E} .
\end{aligned}
$$

Problem 1 (Output regulation with global asymptotic stability of the compensated hybrid dynamics over $\mathscr{T}_{\tau}$ )

Let the hybrid plant $\Sigma_{P}$, the hybrid exogenous system $\Sigma_{E}$, and the positive real constant $\tau$ be given. Find a hybrid feedback regulator $\Sigma_{R}$ such that the hybrid closed-loop system $\Sigma_{L}$ satisfies the following requirements:

$\mathcal{R} 1$. the regulation error $e_{R}(t)$ goes to 0 as $t$ approaches $\infty$, for all the initial states $x_{L}(0)=x_{L 0}$ and all the exogenous signals $x_{E}(t)$ generated by $\Sigma_{E}$,

$\mathcal{R} 2$. the hybrid linear dynamics of $\Sigma_{L}$ is globally asymptotically stable,

for all $\mathcal{T} \in \mathscr{T}_{\tau}$.

It is just worth mentioning that the output regulation problem presented above refers to a measurement feedback compensation scheme. The simpler and more common error feedback compensation scheme can be retrieved as the special case of the former where $e_{R}(t)=e_{M}(t)$, or equivalently, in the equations of $\Sigma_{P}$ and $\Sigma_{E}, E_{P}=C_{P}, D_{P}=0$, and $E_{E}=C_{E}$. 


\section{AN IMPLICIT SUFFICIENT CONDITION}

In this section, a sufficient condition for a hybrid feedback regulator $\Sigma_{R}$ to solve Problem 1 will be expressed with reference to the so-called hybrid autonomous system - i.e., the connection between the hybrid closed-loop system $\Sigma_{L}$ and the hybrid exogenous system $\Sigma_{E}$, described by

$$
\hat{\Sigma} \equiv\left\{\begin{aligned}
\dot{\hat{x}}(t) & =\hat{A} \hat{x}(t), \quad t \in \mathbb{R}^{+} \backslash \mathcal{T}, \\
\hat{x}\left(t_{k}\right) & =\hat{J} \hat{x}^{-}\left(t_{k}\right), \quad t_{k} \in \mathcal{T}, \\
e(t) & =\hat{E} \hat{x}(t), \quad t \in \mathbb{R}^{+},
\end{aligned}\right.
$$

where

$$
\hat{A}=\left[\begin{array}{cc}
A_{L} & B_{L} \\
0 & A_{E}
\end{array}\right], \quad \hat{J}=\left[\begin{array}{cc}
J_{L} & K_{L} \\
0 & J_{E}
\end{array}\right], \quad \hat{E}=\left[\begin{array}{cc}
E_{L} & D_{L}
\end{array}\right] .
$$

The symbol $\hat{\mathcal{X}}$ denotes the state space of $\hat{\Sigma}$. The symbol $\hat{\mathcal{E}}$ stands for Ker $\hat{E}$.

Since the to-be-designed matrices of the regulator $\Sigma_{R}$ are included in those of the hybrid autonomous system $\hat{\Sigma}$ (in fact, they are hidden in those of the hybrid closed-loop system $\Sigma_{L}$ ), the sufficient condition that will be presented in Theorem 1 is referred to as the implicit sufficient condition. As mentioned earlier, the purpose of such condition is twofold: i) providing insight into how the motion of the hybrid autonomous system may guarantee that all the design requirements are met; ii) paving the way to the proof of a constructive sufficient condition.

A key role in the sufficient condition that will be stated in Theorem 1 is played by the notion of hybrid invariance - henceforth characterized by the symbol $\mathscr{H}$. For the sake of immediacy, the definition of $\mathscr{H}$-invariant subspace and the necessary and sufficient condition for a subspace to be $\mathscr{H}$-invariant will refer to the hybrid autonomous system $\hat{\Sigma}$. However, it is understood that the special inner structure of $\hat{A}$ and $\hat{J}$ shown in (6) does not play any role in these statements.

A subspace $\hat{\mathcal{V}} \subseteq \hat{\mathcal{X}}$ is said to be an $\mathscr{H}$-invariant subspace of $\hat{\Sigma}$ if $\hat{A} \hat{\mathcal{V}} \subseteq \hat{\mathcal{V}}$ and $\hat{J} \hat{\mathcal{V}} \subseteq \hat{\mathcal{V}}$.

A subspace $\hat{\mathcal{V}} \subseteq \hat{\mathcal{X}}$, with a basis matrix $\hat{V}$, is an $\mathscr{H}$-invariant subspace of $\hat{\Sigma}$ if and only if there exist matrices $L_{\hat{A}}$ and $L_{\hat{J}}$, such that

$$
\begin{aligned}
\hat{A} \hat{V} & =\hat{V} L_{\hat{A}}, \\
\hat{J} \hat{V} & =\hat{V} L_{\hat{J}} .
\end{aligned}
$$

It is worth noting that, since $\hat{V}$ is full-rank, the matrices $L_{\hat{A}}, L_{\hat{J}}$ such that (7), (8) hold are unique.

The notion of $\mathscr{H}$-invariance of a subspace $\hat{\mathcal{V}} \subseteq \hat{\mathcal{X}}$ leads to those of hybrid linear dynamics restricted to $\hat{\mathcal{V}}$ and hybrid linear dynamics induced on the quotient space $\hat{\mathcal{X}} / \hat{\mathcal{V}}$. To this aim, note that, by applying a state-space basis transformation $\hat{T}=\left[\hat{V} \hat{T}_{2}\right]$, where $\hat{V}$ is a basis matrix of the $\mathscr{H}$-invariant subspace $\hat{\mathcal{V}}$ and $\hat{T}_{2}$ is any matrix such that $\hat{T}$ is invertible, one gets that the flow and jump dynamic matrices of the hybrid linear system $\hat{\Sigma}$, in the new coordinates, respectively have the 
structure

$$
\hat{A}^{\prime}=\hat{T}^{-1} \hat{A} \hat{T}=\left[\begin{array}{cc}
\hat{A}_{11}^{\prime} & \hat{A}_{12}^{\prime} \\
0 & \hat{A}_{22}^{\prime}
\end{array}\right], \quad \hat{J}^{\prime}=\hat{T}^{-1} \hat{J} \hat{T}=\left[\begin{array}{cc}
\hat{J}_{11}^{\prime} & \hat{J}_{12}^{\prime} \\
0 & \hat{J}_{22}^{\prime}
\end{array}\right] .
$$

The structural zero submatrices in $\hat{A}^{\prime}$ and $\hat{J}^{\prime}$ show that the hybrid linear dynamics of $\hat{\Sigma}$ defines a hybrid linear dynamics restricted to $\hat{\mathcal{V}}$ - from now on denoted by $\left.\hat{\Sigma}\right|_{\hat{\mathcal{V}}}$ - and a hybrid linear dynamics induced on $\hat{\mathcal{X}} / \hat{\mathcal{V}}$ - denoted by $\left.\hat{\Sigma}\right|_{\hat{\mathcal{X}} / \hat{\mathcal{V}}}$

Then, the definitions of inner and outer global asymptotic stability over $\mathscr{T}_{\tau}$ of an $\mathscr{H}$-invariant subspace can be stated as follows.

\section{Definition 1}

Given the positive real constant $\tau$, an $\mathscr{H}$-invariant subspace $\hat{\mathcal{V}} \subseteq \hat{\mathcal{X}}$ of the hybrid linear system $\hat{\Sigma}$ is said to be inner globally asymptotically stable over $\mathscr{T}_{\tau}$ if the hybrid linear dynamics restricted to $\hat{\mathcal{V}}$ — i.e., $\left.\hat{\Sigma}\right|_{\hat{\mathcal{V}}}$ — is globally asymptotically stable for all $\mathcal{T} \in \mathscr{T}_{\tau}$. The subspace $\hat{\mathcal{V}}$ is said to be outer globally asymptotically stable over $\mathscr{T}_{\tau}$ if the hybrid linear dynamics induced on $\hat{\mathcal{X}} / \hat{\mathcal{V}}$ - i.e., $\left.\hat{\Sigma}\right|_{\hat{\mathcal{X}} / \hat{\mathcal{V}}}$ — is globally asymptotically stable for all $\mathcal{T} \in \mathscr{T}_{\tau}$.

In order to state the sufficient condition for the solution of Problem 1 referred to $\hat{\Sigma}$, the subspace $\hat{\mathcal{P}} \subseteq \hat{\mathcal{X}}$ is defined by

$$
\hat{\mathcal{P}}=\operatorname{Im} \hat{P}=\operatorname{Im}\left[\begin{array}{l}
I \\
0
\end{array}\right]
$$

where the partition considered in (10) is consistent with that shown in (6). The subspace $\hat{\mathcal{P}}$ is an $\mathscr{H}$-invariant subspace of the hybrid linear system $\hat{\Sigma}$, since (7), (8) hold with $\hat{V}=\hat{P}, L_{\hat{A}}=A_{L}$, and $L_{\hat{J}}=J_{L}$. From these considerations and the direct inspection of the matrices $\hat{A}$ and $\hat{J}$, it ensues that the hybrid linear dynamics of $\hat{\Sigma}$ restricted to $\hat{\mathcal{P}}$ - i.e., $\left.\hat{\Sigma}\right|_{\hat{\mathcal{P}}}$ - matches the hybrid linear dynamics of the closed-loop system $\Sigma_{L}$, while the hybrid linear dynamics induced by $\hat{\Sigma}$ on $\hat{\mathcal{X}} / \hat{\mathcal{P}}-$ i.e., $\left.\hat{\Sigma}\right|_{\hat{\mathcal{X}} / \hat{\mathcal{P}}}$ — coincides with the hybrid linear dynamics of the exogenous system $\Sigma_{E}$.

Another notion needed to prove the implicit sufficient condition is that of unobservable subspace of a hybrid linear system. Like all the notions previously introduced, also this one is defined with reference to $\hat{\Sigma}$. The unobservable subspace of $\hat{\Sigma}$ - from now on denoted by $\hat{\mathcal{Q}}$ - is the set of all the initial states that give rise to free state motions corresponding to zero output for all $\mathcal{T} \in \mathscr{T}_{\tau}$. A computationally effective characterization of the subspace $\hat{\mathcal{Q}}$ is given by the following lemma.

\section{Lemma 1}

The unobservable subspace $\hat{\mathcal{Q}}$ of the hybrid linear system $\hat{\Sigma}$ is the maximal $\mathscr{H}$-invariant subspace contained in the unobservable subspace of the flow dynamics - this latter subspace being given by $\max \mathcal{J}(\hat{A}, \hat{\mathcal{E}})$ and denoted by $\hat{\mathcal{O}}$.

\section{Proof}

First, note that, since $\mathcal{T}$ is any finite or countably infinite sequence in $\mathscr{T}_{\tau}$, with $\tau$ given, the 
unobservable subspace $\hat{\mathcal{Q}}$ is a locus of trajectories, contained in the kernel $\hat{\mathcal{E}}$ of the output distribution matrix $\hat{E}$, for both the hybrid dynamics and the sole flow dynamics of the hybrid linear system $\hat{\Sigma}$. As a locus of trajectories of the hybrid dynamics, $\hat{\mathcal{Q}}$ is invariant under the linear map $\hat{J} e^{\hat{A} t}$ for all given $t \geq \tau$ : i.e.,

$$
\hat{J} e^{\hat{A} t} \hat{\mathcal{Q}} \subseteq \hat{\mathcal{Q}}
$$

holds for all given $t \geq \tau$. Moreover, as a locus of trajectories of the flow dynamics, $\hat{\mathcal{Q}}$ is invariant under the linear map $\hat{A}$ : i.e.,

$$
\hat{A} \hat{\mathcal{Q}} \subseteq \hat{\mathcal{Q}}
$$

By applying the state space basis transformation $\hat{T}=\left[\hat{T}_{1} \hat{T}_{2}\right]$, where $\hat{T}_{1}$ is a basis matrix of $\hat{\mathcal{Q}}$ and $\hat{T}_{2}$ is any matrix such that $\hat{T}$ is invertible, one gets $\hat{A}^{\prime}$ as in (9) and

$$
\hat{J}^{\prime}=\hat{T}^{-1} \hat{J} \hat{T}=\left[\begin{array}{ll}
\hat{J}_{11}^{\prime} & \hat{J}_{12}^{\prime} \\
\hat{J}_{21}^{\prime} & \hat{J}_{22}^{\prime}
\end{array}\right],
$$

where the structural zero submatrix at the lower left corner of $\hat{A}^{\prime}$, shown in (9), is due to (12). By taking (9) and (13) into account, one gets that

$$
\hat{J}^{\prime} e^{\hat{A}^{\prime} t}=\left[\begin{array}{cc}
\hat{J}_{11}^{\prime} e^{\hat{A}_{11}^{\prime} t} & * \\
\hat{J}_{21}^{\prime} e^{\hat{A}_{11}^{\prime} t} & *
\end{array}\right],
$$

holds for all given $t \geq \tau$, where the symbol ' $*$ ' is used in place of submatrices whose expression is of no special interest to this discussion. By virtue of (11), from (14) it follows that

$$
\hat{J}_{21}^{\prime} e^{\hat{A}_{11}^{\prime} t}=0
$$

holds for all given $t \geq \tau$. Since $e^{\hat{A}_{11}^{\prime} t}$ is nonzero for all $t \in \mathbb{R},(15)$ holds if and only if $\hat{J}_{21}^{\prime}=0$, which, in light of (13), is equivalent to $\hat{J}$-invariance of $\hat{\mathcal{Q}}$. Consequently, $\hat{\mathcal{Q}}$, being both $\hat{A}$-invariant and $\hat{J}$-invariant, is an $\mathscr{H}$-invariant subspace of the hybrid linear system $\hat{\Sigma}$. Moreover, $\hat{\mathcal{Q}}$, as an $\mathscr{H}$-invariant subspace of $\hat{\Sigma}$ contained in $\hat{\mathcal{E}}$, is contained in $\hat{\mathcal{O}}$, since the latter is the maximal $\hat{A}$-invariant subspace contained in $\hat{\mathcal{E}}$. Finally, maximality of $\hat{\mathcal{Q}}$ can be shown by contradiction.

Hence, the implicit sufficient condition for a hybrid linear regulator $\Sigma_{R}$ to solve Problem 1 is stated as follows.

\section{Theorem 1}

Let the hybrid plant $\Sigma_{P}$, the hybrid exogenous system $\Sigma_{E}$, and the positive real constant $\tau$ be given. Then, a hybrid feedback regulator $\Sigma_{R}$ solves Problem 1 if there exists an $\mathscr{H}$-invariant subspace $\hat{\mathcal{V}} \subseteq \hat{\mathcal{X}}$ of the hybrid autonomous system $\hat{\Sigma}$, such that

C 1. $\hat{\mathcal{V}} \oplus \hat{\mathcal{P}}=\hat{\mathcal{X}}$ 
C2. $\hat{\mathcal{V}} \subseteq \hat{\mathcal{E}}$

C 3. $\hat{\mathcal{V}}$ is outer globally asymptotically stable over $\mathscr{T}_{\tau}$.

Proof

First, it will be shown that Requirement $\mathcal{R} 1$ of Problem 1 is satisfied. By virtue of Condition $\mathcal{C} 1$, any state $\hat{x}_{0} \in \hat{\mathcal{X}}$ can be written as $\hat{x}_{0}=\hat{x}_{\hat{\mathcal{V}} 0}+\hat{x}_{\hat{\mathcal{P}} 0}$, where $\hat{x}_{\hat{\mathcal{V}} 0} \in \hat{\mathcal{V}}$ and $\hat{x}_{\hat{\mathcal{P}} 0} \in \hat{\mathcal{P}}$ are unique. Then, consider the free state motion originating from $\hat{x}_{0}$. By virtue of $\mathscr{H}$-invariance of $\hat{\mathcal{V}}$ and $\hat{\mathcal{P}}$, the free motions originating from $\hat{x}_{\hat{\mathcal{V}} 0}$ and $\hat{x}_{\hat{\mathcal{P}} 0}$ respectively evolve on $\hat{\mathcal{V}}$ and $\hat{\mathcal{P}}$. Hence, at the jump time $t_{k} \in \mathcal{T}$, the state is $\hat{x}\left(t_{k}\right)=\hat{x}_{\hat{\mathcal{V}}}\left(t_{k}\right)+\hat{x}_{\hat{\mathcal{P}}}\left(t_{k}\right)$, where

$$
\begin{aligned}
& \hat{x}_{\hat{\mathcal{V}}}\left(t_{k}\right)=\hat{J} e^{\hat{A}\left(t_{k}-t_{k-1}\right)} \cdots \hat{J} e^{\hat{A}\left(t_{1}-t_{0}\right)} \hat{J} e^{\hat{A} t_{0}} \hat{x}_{\hat{\mathcal{V}} 0}, \\
& \hat{x}_{\hat{\mathcal{P}}}\left(t_{k}\right)=\hat{J} e^{\hat{A}\left(t_{k}-t_{k-1}\right)} \cdots \hat{J} e^{\hat{A}\left(t_{1}-t_{0}\right)} \hat{J} e^{\hat{A} t_{0}} \hat{x}_{\hat{\mathcal{P}} 0} .
\end{aligned}
$$

Moreover, at the time $t \in\left[t_{k}, t_{k+1}\right)$, the state is $\hat{x}(t)=\hat{x}_{\hat{\mathcal{V}}}(t)+\hat{x}_{\hat{\mathcal{P}}}(t)$, where $\hat{x}_{\hat{\mathcal{V}}}(t)=e^{\hat{A}\left(t-t_{k}\right)} \hat{x}_{\hat{\mathcal{V}}}\left(t_{k}\right)$ and $\hat{x}_{\hat{\mathcal{P}}}(t)=e^{\hat{A}\left(t-t_{k}\right)} \hat{x}_{\hat{\mathcal{P}}}\left(t_{k}\right)$. So, let us consider these two motions separately. Concerning the motion on $\hat{\mathcal{V}}$, this gives rise to zero output for all $t \in \mathbb{R}^{+}$, since $\hat{\mathcal{V}} \subseteq \hat{\mathcal{Q}}$. In fact, $\hat{\mathcal{V}}$, as an $\hat{A}$-invariant subspace contained in $\hat{\mathcal{E}}$ (Condition $\mathcal{C} 2$ ), is contained in $\hat{\mathcal{O}}$. Moreover, as an $\mathscr{H}$-invariant subspace contained in $\hat{\mathcal{O}}$, it is contained in $\hat{\mathcal{Q}}$. As to the free motion on $\hat{\mathcal{P}}$, this converges to the origin as the time approaches infinity (and so does the output), since the hybrid linear dynamics of $\hat{\Sigma}$ restricted to $\hat{\mathcal{P}}$ is globally asymptotically stable over $\mathscr{T}_{\tau}$. In fact, by virtue of Condition $\mathcal{C} 1$, the hybrid linear dynamics of $\hat{\Sigma}$ restricted to $\hat{\mathcal{P}}$ coincides with the hybrid linear dynamics induced by $\hat{\Sigma}$ on $\hat{\mathcal{X}} / \hat{\mathcal{V}}$. Moreover, the latter is globally asymptotically stable over $\mathscr{T}_{\tau}$ by Condition $\mathcal{C} 3$. This completes the proof that Requirement $\mathcal{R} 1$ is met.

As to the proof that also Requirement $\mathcal{R} 2$ is satisfied, it suffices to notice that, as pointed out after the definition of the subspace $\hat{\mathcal{P}}$, the hybrid linear dynamics of the closed-loop system $\Sigma_{L}$ matches the hybrid dynamics of $\hat{\Sigma}$ restricted to $\hat{\mathcal{P}}$ and the latter, as mentioned above, is globally asymptotically stable over $\mathscr{T}_{\tau}$ by Condition $\mathcal{C} 3$.

As mentioned above, in the sufficient condition for the solution of Problem 1 established by Theorem 1, the matrices of the hybrid feedback regulator are contained in those of the hybrid autonomous system. The aim of the next section is to provide a new sufficient condition, expressed in terms of structural and stabilizability properties of a certain subspace, defined for the system consisting of the interconnection between the plant and the exogenous system only. In this way, if such condition - which is called the explicit sufficient condition - holds, the matrices of the feedback regulator can be derived from those of the plant and of the exogenous system. 


\section{AN EXPLICIT SUFFICIENT CONDITION}

This section shows a new sufficient condition for the solution of the output regulation problem with global asymptotic stability, based on the so-called extended hybrid system — namely, the connection between the hybrid plant $\Sigma_{P}$ and the hybrid exogenous system $\Sigma_{E}$ described by

$$
\Sigma \equiv\left\{\begin{aligned}
\dot{x}(t) & =A x(t)+B u(t), \quad t \in \mathbb{R}^{+} \backslash \mathcal{T}, \\
x\left(t_{k}\right) & =J x^{-}\left(t_{k}\right), \quad t_{k} \in \mathcal{T}, \\
e_{R}(t) & =E x(t)+D u(t), \quad t \in \mathbb{R}^{+} \\
e_{M}(t) & =C x(t), \quad t \in \mathbb{R}^{+},
\end{aligned}\right.
$$

where

$$
\begin{aligned}
& A=\left[\begin{array}{cc}
A_{P} & H_{P} \\
0 & A_{E}
\end{array}\right], \quad B=\left[\begin{array}{c}
B_{P} \\
0
\end{array}\right], \quad J=\left[\begin{array}{cc}
J_{P} & K_{P} \\
0 & J_{E}
\end{array}\right], \\
& E=\left[\begin{array}{cc}
-E_{P} & E_{E}
\end{array}\right], \quad D=-D_{P}, \quad C=\left[\begin{array}{cc}
-C_{P} & C_{E}
\end{array}\right] .
\end{aligned}
$$

The state space of $\Sigma$ is denoted by $\mathcal{X}$. The subspace $\operatorname{Im} B$ is denoted by $\mathcal{B}$. The algebraic link from the control input $u$ to the regulated output $e_{R}$ is called the feedthrough term.

In order to express the explicit sufficient condition for problem solvability, the notion of $\mathscr{H}$-controlled invariant subspace of a hybrid linear system with a control input is first defined and then characterized by a couple of necessary and sufficient conditions. To the same purpose, the notion of output-nulling $\mathscr{H}$-controlled invariant subspace of a hybrid linear system which exhibits a feedthrough term is also defined and then characterized by two necessary and sufficient conditions. For ease of reference, these statements will directly address the hybrid linear system $\Sigma$. Consequently, the output-nulling $\mathscr{H}$-controlled invariant subspace will be defined with reference to the regulated output, which is the one affected by the feedthrough term. Nevertheless, it is understood that the special inner structure of the matrices $A, B, J, E$, and $D$ shown in (16)-(17) does not play any part in such definitions and properties.

A subspace $\mathcal{V} \subseteq \mathcal{X}$ is said to be an $\mathscr{H}$-controlled invariant subspace of the hybrid linear system $\Sigma$ if

$$
\begin{aligned}
& A \mathcal{V} \subseteq \mathcal{V}+\mathcal{B}, \\
& J \mathcal{V} \subseteq \mathcal{V} .
\end{aligned}
$$

A subspace $\mathcal{V} \subseteq \mathcal{X}$, with a basis matrix $V$, is an $\mathscr{H}$-controlled invariant subspace of $\Sigma$ if and only if there exist matrices $L_{A}, L_{J}$, and $M$, such that

$$
\begin{aligned}
& A V=V L_{A}+B M, \\
& J V=V L_{J} .
\end{aligned}
$$


Moreover, a subspace $\mathcal{V} \subseteq \mathcal{X}$ is an $\mathscr{H}$-controlled invariant subspace of $\Sigma$ if and only if there exists a linear map $F: \mathcal{X} \rightarrow \mathcal{U}$ such that

$$
(A+B F) \mathcal{V} \subseteq \mathcal{V}
$$

holds along with (19). Any such $F$ is said to be a friend of the $\mathscr{H}$-controlled invariant subspace $\mathcal{V}$. The first characterization of $\mathcal{V}$ as an $\mathscr{H}$-controlled invariant subspace of the hybrid linear system $\Sigma$ expresses the notion of simultaneous $(A, \mathcal{B})$-controlled invariance and $J$-invariance in a coordinatedependent setting. The second characterization, which is coordinate-free, is shown to be equivalent to the former via the coordinate-dependent characterization of $(A+B F)$-invariance: i.e., it ensues from $(A+B F) V=V L_{A}$ by picking $F$ such that $F V=-M$.

The following definition introduces the notion of output-nulling $\mathscr{H}$-controlled invariant subspace of the hybrid linear system $\Sigma$ and, as mentioned above, refers to the regulated output $e_{R}$. Then, two necessary and sufficient conditions for a subspace to be an output-nulling $\mathscr{H}$-controlled invariant subspace of $\Sigma$ follow.

\section{Definition 2}

A subspace $\mathcal{V} \subseteq \mathcal{X}$ is said to be an output-nulling $\mathscr{H}$-controlled invariant subspace of the hybrid linear system $\Sigma$ if it is an $\mathscr{H}$-controlled invariant subspace of $\Sigma$ and there exists a linear map $F: \mathcal{X} \rightarrow \mathcal{U}$ such that (22) holds along with

$$
\mathcal{V} \subseteq \operatorname{Ker}(E+D F)
$$

Any such $F$ is said to be a friend of $\mathcal{V}$.

A subspace $\mathcal{V} \subseteq \mathcal{X}$, with a basis matrix $V$, is an output-nulling $\mathscr{H}$-controlled invariant subspace of the hybrid linear system $\Sigma$ if and only if there exist matrices $L_{A}, L_{J}$, and $F$, such that

$$
\begin{aligned}
& (A+B F) V=V L_{A} \\
& (E+D F) V=0
\end{aligned}
$$

hold along with (21). Moreover, a subspace $\mathcal{V} \subseteq \mathcal{X}$, with a basis matrix $V$, is an output-nulling $\mathscr{H}$-controlled invariant subspace of the hybrid linear system $\Sigma$ if and only if there exist matrices $L_{A}, L_{J}$, and $M$, such that

$$
E V=D M
$$

holds along with (20) and (21). In fact, (20) and (26) can be written as (24) and (25) by picking $F$ such that $F V=-M$.

In order to state Theorem 2, which establishes the explicit sufficient condition, as well as Lemma 2, which paves the way to its proof, the subspace $\mathcal{P} \subseteq \mathcal{X}$ is defined as

$$
\mathcal{P}=\operatorname{Im} P=\operatorname{Im}\left[\begin{array}{l}
I \\
0
\end{array}\right],
$$


where the partition of the basis matrix $P$ shown in (27) is consistent with the partition considered in (16)-(17). The subspace $\mathcal{P}$ is an $\mathscr{H}$-invariant subspace of the hybrid extended system $\Sigma$. In fact, (20) and (21) hold with $V=P, L_{A}=A_{P}, M=0$, and $L_{J}=J_{P}$. As a consequence of $\mathscr{H}$-invariance of $\mathcal{P}$, the hybrid linear dynamics of $\Sigma$ defines a hybrid linear dynamics restricted to $\mathcal{P}$ - namely, $\left.\Sigma\right|_{\mathcal{P}}$ — and a hybrid linear dynamics induced on $\mathcal{X} / \mathcal{P}$ - i.e., $\left.\Sigma\right|_{\mathcal{X} / \mathcal{P}}$. In particular, from the upper blocktriangular structure of $A$ and $J$ shown in (16), it ensues that the restricted dynamics $\left.\Sigma\right|_{\mathcal{P}}$ matches the hybrid linear dynamics of the plant $\Sigma_{P}$, while the induced dynamics $\left.\Sigma\right|_{\mathcal{X} / \mathcal{P}}$ corresponds to the hybrid linear dynamics of the exogenous system $\Sigma_{E}$.

\section{Lemma 2}

Let $\mathcal{V} \subseteq \mathcal{X}$ be an output-nulling $\mathscr{H}$-controlled invariant subspace of the hybrid linear system $\Sigma$. Let $\mathcal{V}$ be such that

$$
\mathcal{V} \oplus \mathcal{P}=\mathcal{X}
$$

Let a basis matrix $V$ of $\mathcal{V}$ be partitioned as

$$
V=\left[\begin{array}{c}
V_{P} \\
I
\end{array}\right]
$$

according to (16)-(17). Let a friend $F: \mathcal{X} \rightarrow \mathcal{U}$ of $\mathcal{V}$ be accordingly partitioned as

$$
F=\left[\begin{array}{ll}
F_{P} & F_{E}
\end{array}\right] \text {. }
$$

Then, the linear map $F^{\prime}: \mathcal{X} \rightarrow \mathcal{U}$ defined by

$$
F^{\prime}=\left[\begin{array}{ll}
F_{P}+X & F_{E}-X V_{P}
\end{array}\right]
$$

where $V_{P}, F_{P}, F_{E}$ are as in (29), (30) and $X$ is any real matrix of appropriate dimensions, is such that $\left(A+B F^{\prime}\right) \mathcal{V} \subseteq \mathcal{V}$ and $\mathcal{V} \subseteq \operatorname{Ker}\left(E+D F^{\prime}\right)$ hold.

\section{Proof}

First, note that there is no loss of generality in assuming (29) as a basis matrix of $\mathcal{V}$, since $\mathcal{V}$ is a direct summand of $\mathcal{P}$ by assumption. Then, (22) is equivalent to the existence of a matrix $L_{F}$ such that

$$
\left[\begin{array}{cc}
A_{P}+B_{P} F_{P} & H_{P}+B_{P} F_{E} \\
0 & A_{E}
\end{array}\right]\left[\begin{array}{c}
V_{P} \\
I
\end{array}\right]=\left[\begin{array}{c}
V_{P} \\
I
\end{array}\right] L_{F}
$$

holds, where (16), (29), and (30) have been taken into account. In particular, (32) holds with $L_{F}=A_{E}$ and, therefore, it is equivalent to

$$
\left(A_{P}+B_{P} F_{P}\right) V_{P}+\left(H_{P}+B_{P} F_{E}\right)=V_{P} A_{E} .
$$

A similar reasoning shows that $\left(A+B F^{\prime}\right) \mathcal{V} \subseteq \mathcal{V}$ holds if and only if there exists $L_{F}^{\prime}$ such that

$$
\left[\begin{array}{cc}
A_{P}+B_{P} F_{P}+B_{P} X & H_{P}+B_{P} F_{E}-B_{P} X V_{P} \\
0 & A_{E}
\end{array}\right]\left[\begin{array}{c}
V_{P} \\
I
\end{array}\right]=\left[\begin{array}{c}
V_{P} \\
I
\end{array}\right] L_{F}^{\prime}
$$


holds, where (16), (29), and (31) have been considered. By virtue of (33), (34) holds with $L_{F}^{\prime}=A_{E}$. Moreover, (23) is equivalent to $(E+D F) V=0$, which can be written as

$$
\left[\begin{array}{cc}
-E_{P}-D_{P} F_{P} & E_{E}-D_{P} F_{E}
\end{array}\right]\left[\begin{array}{c}
V_{P} \\
I
\end{array}\right]=0
$$

where (17) and (29) have been taken into consideration. Further, (35) can be written as

$$
-\left(E_{P}+D_{P} F_{P}\right) V_{P}+\left(E_{E}-D_{P} F_{E}\right)=0
$$

Likewise, $\mathcal{V} \subseteq \operatorname{Ker}\left(E+D F^{\prime}\right)$ is equivalent to

$$
-\left(E_{P}+D_{P} F_{P}\right) V_{P}-D_{P} X V_{P}+\left(E_{E}-D_{P} F_{E}\right)+D_{P} X V_{P}=0
$$

which holds by virtue of (36).

It is worth noting that, on the assumption that the $\mathscr{H}$-controlled invariant subspace $\mathcal{V}$ is a direct summand of $\mathcal{P}$ or, equivalently, on the assumption that a basis matrix $V$ of $\mathcal{V}$ has the structure shown in (29), $J$-invariance of $\mathcal{V}$ is equivalent to the existence of a matrix $L_{J}$ such that

$$
\left[\begin{array}{cc}
J_{P} & K_{P} \\
0 & J_{E}
\end{array}\right]\left[\begin{array}{c}
V_{P} \\
I
\end{array}\right]=\left[\begin{array}{c}
V_{P} \\
I
\end{array}\right] L_{J}
$$

holds, where (16) and (29) have been taken into consideration. Consequently, (37) is equivalent to

$$
J_{P} V_{P}+K_{P}=V_{P} J_{E}
$$

Concerning the subspace $\mathcal{P}$, it is also worth noting that, since $\mathcal{B} \subseteq \mathcal{P}$ (compare $B$ shown (16) with $P$ shown in (27)), for any $F: \mathcal{X} \rightarrow \mathcal{U}$, the subspace $\mathcal{P}$ is an $\mathscr{H}$-invariant subspace of the compensated hybrid linear system $\Sigma_{F}$ defined by

$$
\Sigma_{F} \equiv\left\{\begin{aligned}
\dot{x}(t) & =(A+B F) x(t), \quad t \in \mathbb{R}^{+} \backslash \mathcal{T}, \\
x\left(t_{k}\right) & =J x^{-}\left(t_{k}\right), \quad t_{k} \in \mathcal{T}, \\
e_{R}(t) & =(E+D F) x(t), \quad t \in \mathbb{R}^{+} \\
e_{M}(t) & =C x(t), \quad t \in \mathbb{R}^{+} .
\end{aligned}\right.
$$

In fact,

$$
\left[\begin{array}{cc}
A_{P}+B_{P} F_{P} & H_{P}+B_{P} F_{E} \\
0 & A_{E}
\end{array}\right]\left[\begin{array}{l}
I \\
0
\end{array}\right]=\left[\begin{array}{l}
I \\
0
\end{array}\right] L_{F}
$$

holds with $L_{F}=A_{P}+B_{P} F_{P}$. Consequently, the hybrid linear dynamics of $\Sigma_{F}$ restricted to $\mathcal{P}-$ i.e., $\left.\Sigma_{F}\right|_{\mathcal{P}}$ - is well-defined and matches the hybrid linear dynamics of the plant $\Sigma_{P}$ when the state feedback $F_{P}$ - refer to the partition of $F$ shown in (30) - is applied to the flow dynamics.

In light of the previous statements, the explicit sufficient condition for solvability of Problem 1 can be formulated as follows. 
Theorem 2

Let the hybrid plant $\Sigma_{P}$, the hybrid exogenous system $\Sigma_{E}$, and the positive real constant $\tau$ be given. Let $\left(A_{P}, B_{P}\right)$ be reachable and $(A, C)$ be observable. Problem 1 has a solution if there exists an output nulling $\mathscr{H}$-controlled invariant subspace $\mathcal{V} \subseteq \mathcal{X}$ of the extended hybrid system $\Sigma$ such that (28) holds.

Proof

Let $\mathcal{V} \subseteq \mathcal{X}$ be an output-nulling $\mathscr{H}$-controlled invariant subspace of the extended hybrid system $\Sigma$ satisfying (28). Let $F: \mathcal{X} \rightarrow \mathcal{U}$ be a friend of $\mathcal{V}$ such that the restricted compensated hybrid dynamics $\left.\Sigma_{F}\right|_{\mathcal{P}}$ be globally asymptotically stable over $\mathscr{T}_{\tau}$. Note that the two requirements on $F$ are compatible by virtue of Lemma 2. Also note that global asymptotic stability of $\left.\Sigma_{F}\right|_{\mathcal{P}}$ over $\mathscr{T}_{\tau}$ can be achieved by virtue of Proposition 2 in the Appendix, since $\left(A_{P}, B_{P}\right)$ is reachable by assumption. Let $G: \mathcal{Y}_{M} \rightarrow \mathcal{X}$ be such that the compensated hybrid linear system $\Sigma_{G}$, defined by

$$
\Sigma_{G} \equiv\left\{\begin{aligned}
\dot{x}(t) & =(A+G C) x(t), \quad t \in \mathbb{R}^{+} \backslash \mathcal{T}, \\
x\left(t_{k}\right) & =J x^{-}\left(t_{k}\right), \quad t_{k} \in \mathcal{T}, \\
e_{M}(t) & =C x(t), \quad t \in \mathbb{R}^{+},
\end{aligned}\right.
$$

be globally asymptotically stable over $\mathscr{T}_{\tau}$. Note that global asymptotic stability of $\Sigma_{G}$ over $\mathscr{T}_{\tau}$ can be attained by virtue of Proposition 3 in the Appendix, since $(A, C)$ is observable by assumption. Then, it will be shown that the hybrid feedback regulator $\Sigma_{R}$, designed by picking

$$
A_{R}=A+B F+G C, \quad B_{R}=-G, \quad J_{R}=J, \quad C_{R}=F, \quad D_{R}=0,
$$

with $F$ and $G$ defined as specified above, solves Problem 1. First, note that, by replacing (39) in (3)-(5), one gets

$$
\begin{aligned}
A_{L} & =\left[\begin{array}{cc}
A_{P} & B_{P} F \\
G C_{P} & A+B F+G C
\end{array}\right], \quad B_{L}=\left[\begin{array}{c}
H_{P} \\
-G C_{E}
\end{array}\right], \\
J_{L} & =\left[\begin{array}{cc}
J_{P} & 0 \\
0 & J
\end{array}\right], \quad K_{L}=\left[\begin{array}{c}
K_{P} \\
0
\end{array}\right], \\
E_{L} & =\left[\begin{array}{cc}
-E_{P} & -D_{P} F
\end{array}\right], \quad D_{L}=E_{E} .
\end{aligned}
$$

Then, by replacing (40)-(42) in (6), one gets

$$
\begin{aligned}
& \hat{A}=\left[\begin{array}{ccc}
A_{P} & B_{P} F & H_{P} \\
G C_{P} & A+B F+G C & -G C_{E} \\
0 & 0 & A_{E}
\end{array}\right], \quad \hat{J}=\left[\begin{array}{ccc}
J_{P} & 0 & K_{P} \\
0 & J & 0 \\
0 & 0 & J_{E}
\end{array}\right], \\
& \hat{E}=\left[\begin{array}{ccc}
-E_{P} & -D_{P} F & E_{E}
\end{array}\right] .
\end{aligned}
$$


Moreover, by applying the state space basis transformation

$$
\hat{T}=\left[\begin{array}{lll}
I & 0 & 0 \\
0 & 0 & I \\
0 & I & 0
\end{array}\right]
$$

one gets

$$
\begin{aligned}
& \hat{A}^{\prime}=\hat{T}^{-1} \hat{A} \hat{T}=\left[\begin{array}{ccc}
A_{P} & H_{P} & B_{P} F \\
0 & A_{E} & 0 \\
G C_{P} & -G C_{E} & A+B F+G C
\end{array}\right], \\
& \hat{J}^{\prime}=\hat{T}^{-1} \hat{J} \hat{T}=\left[\begin{array}{ccc}
J_{P} & K_{P} & 0 \\
0 & J_{E} & 0 \\
0 & 0 & J
\end{array}\right], \\
& \hat{E}^{\prime}=\hat{E} \hat{T}=\left[\begin{array}{lll}
-E_{P} & E_{E} & -D_{P} F
\end{array}\right] .
\end{aligned}
$$

By taking (16)-(17) into account, one can write (46)-(48) in more compact form as

$$
\begin{aligned}
\hat{A}^{\prime} & =\left[\begin{array}{cc}
A & B F \\
-G C & A+B F+G C
\end{array}\right], \\
\hat{J}^{\prime} & =\left[\begin{array}{ll}
J & 0 \\
0 & J
\end{array}\right] \\
\hat{E}^{\prime} & =\left[\begin{array}{ll}
E & D F
\end{array}\right] .
\end{aligned}
$$

Hence, it will be shown that the subspace $\hat{\mathcal{V}}=\operatorname{Im} \hat{V}^{\prime}$, where

$$
\hat{V}^{\prime}=\left[\begin{array}{c}
V \\
V
\end{array}\right],
$$

with $V$ denoting a basis matrix of $\mathcal{V}$ defined as in (29), is an $\mathscr{H}$-invariant subspace of the hybrid autonomous system $\hat{\Sigma}$, satisfying Conditions $\mathcal{C} 1-\mathcal{C} 3$ of Theorem 1. First, note that (7), where $\hat{A}$ is replaced by $\hat{A}^{\prime}$ given by (49) and $\hat{V}$ is replaced by $\hat{V}^{\prime}$ given by (52), holds with $L_{\hat{A}}=A_{E}$, by virtue of (16), (29), (30), and (33). Moreover, (8), where $\hat{J}$ and $\hat{V}$ are respectively replaced by $\hat{J}^{\prime}$ and $\hat{V}^{\prime}$ given by (50) and (52), holds with $L_{\hat{J}}=J_{E}$, by virtue of (16), (29), and (38). Thus, it is proven that $\hat{\mathcal{V}}$ is an $\mathscr{H}$-invariant subspace of $\hat{\Sigma}$. In order to show that Condition $\mathcal{C} 1$ of Theorem 1 is satisfied, note that, with respect to the coordinates and the partition considered in (43)-(44), the basis matrix $\hat{P}$ of the subspace $\hat{\mathcal{P}}$, defined by (10), is given by

$$
\hat{P}=\left[\begin{array}{ll}
I & 0 \\
0 & I \\
0 & 0
\end{array}\right] .
$$


Hence, by applying the state space basis transformation $\hat{T}$ defined by (45), one gets

$$
\hat{P}^{\prime}=\hat{T}^{-1} \hat{P}=\left[\begin{array}{cc}
I & 0 \\
0 & 0 \\
0 & I
\end{array}\right],
$$

which, with respect to the partition considered in (49)-(51), can be written as

$$
\hat{P}^{\prime}=\left[\begin{array}{ll}
P & 0 \\
0 & I
\end{array}\right]
$$

where (27) has been taken into account. Therefore, the comparison between the basis matrices $\hat{V}^{\prime}$ and $\hat{P}^{\prime}$ respectively shown in (52) and (53), in light of (28), proves that Condition $\mathcal{C} 1$ of Theorem 1 is fulfilled. To show that Condition $\mathcal{C} 2$ of Theorem 1 is also met, note that

$$
\hat{E}^{\prime} \hat{V}^{\prime}=\left[\begin{array}{ll}
E & D F
\end{array}\right]\left[\begin{array}{c}
V \\
V
\end{array}\right]=(E+D F) V=0,
$$

in light of (51), (52), (17), (29), (30), since (36) holds. In order to prove that Condition $\mathcal{C} 3$ of Theorem 1 is satisfied, first note that $\hat{\mathcal{X}} / \hat{\mathcal{V}}=\hat{\mathcal{P}}$, since, as shown above, $\hat{\mathcal{V}}$ is a direct summand of $\hat{\mathcal{P}}$. Thus, the induced hybrid dynamics $\left.\hat{\Sigma}\right|_{\hat{\mathcal{X}} / \hat{\mathcal{V}}}$ matches the restricted hybrid dynamics $\left.\hat{\Sigma}\right|_{\hat{\mathcal{P}}}$. Consequently, proving that Condition $\mathcal{C} 3$ holds reduces to proving that the hybrid linear dynamics $\left.\hat{\Sigma}\right|_{\hat{\mathcal{P}}}$ is globally asymptotically stable over $\mathscr{T}_{\tau}$ or, in light of the considerations made in Section 3, it reduces to proving that the hybrid dynamics of the closed-loop system $\Sigma_{L}$ is globally asymptotically stable over $\mathscr{T}_{\tau}$. To this aim, a more detailed partition of (40) and (41) is considered, where $F$ is partitioned according to (30) and $G$ is consistently partitioned as

$$
G=\left[\begin{array}{l}
G_{P} \\
G_{E}
\end{array}\right]
$$

Thus, it ensues that

$$
\begin{aligned}
A_{L} & =\left[\begin{array}{ccc}
A_{P} & B_{P} F_{P} & B_{P} F_{E} \\
G_{P} C_{P} & A_{P}+B_{P} F_{P}-G_{P} C_{P} & H_{P}+B_{P} F_{E}+G_{P} C_{E} \\
G_{E} C_{P} & -G_{E} C_{P} & A_{E}+G_{E} C_{E}
\end{array}\right], \\
J_{L} & =\left[\begin{array}{ccc}
J_{P} & 0 & 0 \\
0 & J_{P} & K_{P} \\
0 & 0 & J_{E}
\end{array}\right] .
\end{aligned}
$$

Then, by applying the state space basis transformation

$$
T_{L}=\left[\begin{array}{lll}
I & 0 & 0 \\
I & I & 0 \\
0 & 0 & I
\end{array}\right],
$$


one gets

$$
\begin{aligned}
& A_{L}^{\prime}=T_{L}^{-1} A_{L} T_{L}=\left[\begin{array}{ccc}
A_{P}+B_{P} F_{P} & B_{P} F_{P} & B_{P} F_{E} \\
0 & A_{P}-G_{P} C_{P} & H_{P}+G_{P} C_{E} \\
0 & -G_{E} C_{P} & A_{E}+G_{E} C_{E}
\end{array}\right] \text {, } \\
& J_{L}^{\prime}=T_{L}^{-1} J_{L} T_{L}=\left[\begin{array}{ccc}
J_{P} & 0 & 0 \\
0 & J_{P} & K_{P} \\
0 & 0 & J_{E}
\end{array}\right] \text {. }
\end{aligned}
$$

Hence, by taking (16)-(17), (30), and (55) into account, one can rewrite (56) and (57) in more compact form as

$$
\begin{aligned}
A_{L}^{\prime} & =\left[\begin{array}{cc}
A_{P}+B_{P} F_{P} & B_{P} F \\
0 & A+G E
\end{array}\right], \\
J_{L}^{\prime} & =\left[\begin{array}{cc}
J_{P} & 0 \\
0 & J
\end{array}\right] .
\end{aligned}
$$

The upper block-triangular structure of $A_{L}^{\prime}$ and $J_{L}^{\prime}$ shows that the hybrid dynamics of $\Sigma_{L}$ is the composition of those of $\left.\Sigma_{F}\right|_{\mathcal{P}}$ and $\Sigma_{G}$ and these latter are globally asymptotically stable over $\mathscr{T}_{\tau}$, owing to the special choice of the linear maps $F$ and $G$.

It is worth pointing out that the proof of Theorem 2 shows how to obtain the matrices of the hybrid feedback regulator, given an output-nulling $\mathscr{H}$-controlled invariant subspace $\mathcal{V}$ of the extended hybrid system $\Sigma$, with the property of being a direct summand of $\mathcal{P}-$ such subspace will be called a resolving subspace.

\section{A NECESSARY AND SUFFICIENT CONDITION FOR THE EXISTENCE OF A RESOLVING SUBSPACE}

This section is focused on the computation of an output-nulling $\mathscr{H}$-controlled invariant subspace of the extended hybrid system $\Sigma$, with the property of being a direct summand of $\mathcal{P}$ (i.e., satisfying the condition of Theorem 2). Since no role is played in this regard by the measured output $e_{M}$, this will not be considered hereafter. In particular, a necessary and sufficient condition for the existence of a subspace satisfying the requisites of Theorem 2 will be presented in Theorem 3 . Such characterization exploits a special output-nulling $\mathscr{H}$-controlled invariant subspace, directly computable by an algorithm, and shows how to use it to derive a resolving subspace (if any exists). Thus, the constructive proof of the if-part of Theorem 3 completes the computational procedure for the synthesis of the hybrid feedback regulator outlined in the proof of Theorem 2.

As can be proven by simple arguments of linear algebra, the set of all output-nulling $\mathscr{H}$-controlled invariant subspaces of the extended hybrid system $\Sigma$ is an upper semilattice with 
respect to the sum and the inclusion. Therefore, such set has a maximum, henceforth denoted by $\mathcal{V}_{\mathscr{O} \mathscr{H}}^{*}$, and this will be the key subspace in Theorem 3. The subspace $\mathcal{V}_{\mathscr{O} \mathscr{H}}^{*}$ can be computed through a procedure that hinges on the following Proposition 1 and Algorithm 1 and that can be outlined in these terms: i) the hybrid linear system $\Sigma$, which exhibits a feedthrough term from the control input to the output, is associated to a suitably-defined hybrid linear system $\tilde{\Sigma}$, which does not show any feedthrough term; ii) the algorithm for computing the maximal $\mathscr{H}$-controlled invariant subspace contained in the kernel of the output map is applied to $\tilde{\Sigma}$; iii) the maximal output-nulling $\mathscr{H}$-controlled invariant subspace of $\Sigma$ is derived from the maximal $\mathscr{H}$-controlled invariant subspace contained in the kernel of the output map of $\tilde{\Sigma}$ by exploiting an appropriate relation between the two.

Hence, let the hybrid linear system $\tilde{\Sigma}$ be defined as the cascade connection of the hybrid linear system $\Sigma$ and of an integrator applied to its output: i.e.,

$$
\tilde{\Sigma} \equiv\left\{\begin{aligned}
\dot{\tilde{x}}(t) & =\tilde{A} \tilde{x}(t)+\tilde{B} u(t), \quad t \in \mathbb{R}^{+} \backslash \mathcal{T}, \\
\tilde{x}\left(t_{k}\right) & =\tilde{J} \tilde{x}^{-}\left(t_{k}\right), \quad t_{k} \in \mathcal{T}, \\
\tilde{e}(t) & =\tilde{E} \tilde{x}(t), \quad t \in \mathbb{R}^{+},
\end{aligned}\right.
$$

where

$$
\tilde{A}=\left[\begin{array}{cc}
A & 0 \\
E & 0
\end{array}\right], \quad \tilde{B}=\left[\begin{array}{c}
B \\
D
\end{array}\right], \quad \tilde{J}=\left[\begin{array}{cc}
J & 0 \\
0 & I
\end{array}\right], \quad \tilde{E}=\left[\begin{array}{ll}
0 & I
\end{array}\right] .
$$

The state space of $\tilde{\Sigma}$ is denoted by $\tilde{\mathcal{X}}$. The kernel of $\tilde{E}$ is denoted by $\tilde{\mathcal{E}}$. The set of all $\mathscr{H}$-controlled invariant subspaces of $\tilde{\Sigma}$ contained in $\tilde{\mathcal{E}}$ is an upper semilattice with respect to the sum and the inclusion and its maximum is denoted by $\tilde{\mathcal{V}}_{\mathscr{H}}^{*}$. The following algorithm shows how to derive $\tilde{\mathcal{V}}_{\mathscr{H}}^{*}$.

\section{Algorithm 1}

The maximal $\mathscr{H}$-controlled invariant subspace of the hybrid linear system $\tilde{\Sigma}$ contained in the subspace $\tilde{\mathcal{E}}$ - i.e., $\tilde{\mathcal{V}}_{\mathscr{H}}^{*}-$ is the last term of the sequence $\tilde{\mathcal{V}}_{0}=\tilde{\mathcal{E}}$, $\tilde{\mathcal{V}}_{i}=\tilde{\mathcal{V}}_{i-1} \cap \tilde{A}^{-1}\left(\tilde{\mathcal{V}}_{i-1}+\tilde{\mathcal{B}}\right) \cap \tilde{J}^{-1} \tilde{\mathcal{V}}_{i-1}$, with $i=1, \ldots, k$, where $k \leq n_{P}+n_{E}+q-1$ is the least integer such that $\tilde{\mathcal{V}}_{k+1}=\tilde{\mathcal{V}}_{k}$.

Indeed, the subspaces subsequently generated by Algorithm 1 form a nested sequence of subspaces of $\tilde{\mathcal{X}}$ of nonincreasing dimension. The last term is contained in $\tilde{\mathcal{E}}$ and, as can be proven by induction, it is the maximal subspace contained in $\tilde{\mathcal{E}}$ such that $\tilde{A} \tilde{\mathcal{V}}_{\mathscr{H}}^{*} \subseteq \tilde{\mathcal{V}}_{\mathscr{H}}^{*}+\tilde{\mathcal{B}}$ and $\tilde{J} \tilde{\mathcal{V}}_{\mathscr{H}}^{*} \subseteq \tilde{\mathcal{V}}_{\mathscr{H}}^{*}$.

The relation between the subspace $\tilde{\mathcal{V}}_{\mathscr{H}}^{*}$ (i.e., the maximal $\mathscr{H}$-controlled invariant subspace of $\tilde{\Sigma}$ contained in $\tilde{\mathcal{E}}$ ) and the subspace $\mathcal{V}_{\mathscr{O}}^{*} \mathscr{H}$ (i.e., the maximal output-nulling $\mathscr{H}$-controlled invariant subspace of the original system $\Sigma$ ) is pointed out by the following proposition.

\section{Proposition 1}

Let the hybrid linear system $\Sigma$ be given and let the hybrid linear system $\tilde{\Sigma}$ be defined according to 
(58). Then, the subspace

$$
\tilde{\mathcal{V}}=\operatorname{Im} \tilde{V}=\operatorname{Im}\left[\begin{array}{l}
V \\
0
\end{array}\right],
$$

where the partition of $\tilde{V}$ shown in (59) is consistent with that in (58), is the maximal $\mathscr{H}$-controlled invariant subspace of $\tilde{\Sigma}$ contained in $\tilde{\mathcal{E}}$ (i.e., $\tilde{\mathcal{V}}=\tilde{\mathcal{V}}_{\mathscr{H}}^{*}$ ) if and only if the subspace $\mathcal{V}=\operatorname{Im} V$ is the maximal output-nulling $\mathscr{H}$-controlled invariant subspace of $\Sigma$ (i.e., $\left.\mathcal{V}=\mathcal{V}_{\mathscr{O}}^{*} \mathscr{H}\right)$.

\section{Proof}

First, note that the structural zero block in the basis matrix $\tilde{V}$ of $\tilde{\mathcal{V}}$ shown in (59) is due to the special structure of the output map $\tilde{E}$ shown in (58) and to the condition $\tilde{\mathcal{V}}_{\mathscr{H}}^{*} \subseteq \tilde{\mathcal{E}}$. Hence, it will first be proven that $\tilde{\mathcal{V}}$ is an $\mathscr{H}$-controlled invariant subspace of $\tilde{\Sigma}$ contained in $\tilde{\mathcal{E}}$ if and only if $\mathcal{V}$ is an output-nulling $\mathscr{H}$-controlled invariant subspace of $\Sigma$. The subspace $\tilde{\mathcal{V}}$ is an $\mathscr{H}$-controlled invariant subspace of $\tilde{\Sigma}$ if and only if there exist matrices $L_{A}, L_{J}$, and $M$ such that

$$
\begin{aligned}
\tilde{A} \tilde{V} & =\tilde{V} L_{A}+\tilde{B} M, \\
\tilde{J} \tilde{V} & =\tilde{V} L_{J} .
\end{aligned}
$$

In light of (58) and (59), (60) and (61) are equivalent to (20), (21) and (26), which, in turn, are equivalent to $\mathcal{V}$ being an output-nulling $\mathscr{H}$-controlled invariant subspace of $\Sigma$. Then, maximality of $\tilde{\mathcal{V}}$ guarantees maximality of $\mathcal{V}$ and viceversa, owing to the special structure of the basis matrix $\tilde{V}$ shown in (59).

Hence, the following lemma paves the way to the necessary and sufficient condition for the existence of a subspace $\mathcal{V}$ satisfying the requisites of Theorem 2.

\section{Lemma 3}

Let the hybrid plant $\Sigma_{P}$ and the hybrid exogenous system $\Sigma_{E}$ be given. Consider the extended hybrid system $\Sigma$, the subspace $\mathcal{P}$, and the subspace $\mathcal{V}_{\mathscr{O} \mathscr{H}}^{*}$. Let $F$ be a friend of $\mathcal{V}_{\mathscr{O}}^{*} \mathscr{H}$. Let $\mathcal{V}_{\mathscr{O}}^{*} \mathscr{H}$ be such that

$$
\mathcal{V}_{\mathscr{O} \mathscr{H}}^{*}+\mathcal{P}=\mathcal{X}
$$

Let the state space basis transformation $T=\left[T_{1} T_{2} T_{3}\right]$, where $\operatorname{Im} T_{1}=\mathcal{V}_{\mathscr{O}}^{*} \mathscr{H} \cap \mathcal{P}$, $\operatorname{Im}\left[T_{1} T_{2}\right]=\mathcal{V}_{\mathscr{O} \mathscr{H}}^{*}$, and $\operatorname{Im}\left[T_{1} T_{3}\right]=\mathcal{P}$, be applied. Then, in the new coordinates, it ensues 
that

$$
\begin{aligned}
& A^{\prime}+B^{\prime} F^{\prime}=T^{-1}(A+B F) T=\left[\begin{array}{ccc}
A_{11}^{\prime}+B_{1}^{\prime} F_{1}^{\prime} & A_{12}^{\prime}+B_{1}^{\prime} F_{2}^{\prime} & A_{13}^{\prime}+B_{1}^{\prime} F_{3}^{\prime} \\
O & A_{22}^{\prime} & O \\
O & O & A_{33}^{\prime}+B_{3}^{\prime} F_{3}^{\prime}
\end{array}\right], \\
& J^{\prime}=T^{-1} J T=\left[\begin{array}{ccc}
J_{11}^{\prime} & J_{12}^{\prime} & J_{13}^{\prime} \\
O & J_{22}^{\prime} & O \\
O & O & J_{33}^{\prime}
\end{array}\right] \\
& E^{\prime}+D F^{\prime}=T^{-1}(E+D F) T=\left[\begin{array}{lll}
O & O & E_{3}^{\prime}+D F_{3}^{\prime}
\end{array}\right] .
\end{aligned}
$$

Proof

Note that $\mathscr{H}$-invariance of $\mathcal{P}$ with respect to the hybrid linear system $\Sigma$ and the inclusion $\mathcal{B} \subseteq \mathcal{P}$ account for the zero matrices in the second block of rows of $A^{\prime}+B^{\prime} F^{\prime}$ and $J^{\prime}$, respectively. Moreover, $\mathscr{H}$-invariance of $\mathcal{V}_{\mathscr{O}}^{*} \mathscr{H}$ with respect to the hybrid compensated system $\Sigma_{F}$ accounts for the zero matrices in the last block of rows of $A^{\prime}+B^{\prime} F^{\prime}$ and $J^{\prime}$, respectively. Finally, the inclusion $\mathcal{V}_{\mathscr{O} \mathscr{H}}^{*} \subseteq \operatorname{Ker}(E+D F)$ accounts for the zero matrices in $E^{\prime}+D F^{\prime}$.

\section{Theorem 3}

Let the hybrid plant $\Sigma_{P}$ and the hybrid exogenous system $\Sigma_{E}$ be given. Consider the extended hybrid system $\Sigma$, the subspace $\mathcal{P}$, and the subspace $\mathcal{V}_{\mathscr{O}}^{*} \mathscr{H}$. There exists an output-nulling $\mathscr{H}$-controlled invariant subspace $\mathcal{V}$ of $\Sigma$ such that (28) holds if and only (62) holds and there exist a friend $F: \mathcal{X} \rightarrow \mathcal{U}$ of $\mathcal{V}_{\mathscr{O}}^{*} \mathscr{H}$ and a matrix $X$ of appropriate dimensions such that, with reference to the coordinates introduced in Lemma 3, the following equations are satisfied:

$$
\begin{aligned}
& \left(A_{11}^{\prime}+B_{1}^{\prime} F_{1}^{\prime}\right) X-X A_{22}^{\prime}=-A_{12}^{\prime}-B_{1}^{\prime} F_{2}^{\prime}, \\
& J_{11}^{\prime} X-X J_{22}^{\prime}=-J_{12}^{\prime} .
\end{aligned}
$$

Proof

If. Let (62) hold. Let $F: \mathcal{X} \rightarrow \mathcal{U}$ be a friend of $\mathcal{V}_{\mathscr{O} \mathscr{H}}^{*}$ and $X$ a matrix of appropriate dimensions such that (66) and (67) are satisfied. It will be proven that the subspace

$$
\mathcal{V}=\operatorname{Im} V^{\prime}=\operatorname{Im}\left[\begin{array}{c}
X \\
I \\
O
\end{array}\right],
$$

where the partition considered in (68) is consistent with that shown in (63)-(65), is an outputnulling $\mathscr{H}$-controlled invariant subspace of $\Sigma$ satisfying (28). To this aim, first, note that $\left(A^{\prime}+B^{\prime} F^{\prime}\right) V^{\prime}=V^{\prime} L_{F}$ holds with $L_{F}=A_{22}^{\prime}$, by virtue of (66), which implies that $\mathcal{V}$ is an $(A+B F)$-invariant subspace or, equivalently, an $(A, \mathcal{B})$-controlled invariant subspace. Secondly, note that $J^{\prime} V^{\prime}=V^{\prime} L_{J}$ holds with $L_{J}=J_{22}^{\prime}$, by virtue of (67), which implies that $\mathcal{V}$ is a $J$-invariant 
subspace. Therefore, $\mathcal{V}$ is an $\mathscr{H}$-controlled invariant subspace of the extended hybrid system $\Sigma$. Moreover, $\left(E^{\prime}+D F^{\prime}\right) V^{\prime}=0$, which implies that $\mathcal{V}$ is contained in $\operatorname{Ker}(E+D F)$. Furthermore, the comparison between the basis matrices $V^{\prime}$ and $P^{\prime}$ of $\mathcal{V}$ and $\mathcal{P}$ with respect to the coordinates introduced in Lemma 3 shows that $\mathcal{V}$ is a direct summand of $\mathcal{P}$.

Only if. Let $\mathcal{V}$ be an output-nulling $\mathscr{H}$-controlled invariant subspace of $\Sigma$, such that (28) holds. Then, all the more reasons for $\mathcal{V}_{\mathscr{O}}^{*} \mathscr{H}$ to satisfy (62). Let $F: \mathcal{X} \rightarrow \mathcal{U}$ be a friend of both $\mathcal{V}$ and $\mathcal{V}_{\mathscr{O}}^{*} \mathscr{H}$. Note that the existence of such a friend $F$ is guaranteed by $\mathcal{V} \subseteq \mathcal{V}_{\mathscr{O}}^{*} \mathscr{H}$, in light of Proposition 1 and [33, Property 4.1-5]. Also note that (28) and $\mathcal{V} \subseteq \mathcal{V}_{\mathscr{O}}^{*} \mathscr{H}$ imply that $\left(\mathcal{V}_{\mathscr{O}}^{*} \mathscr{H} \cap \mathcal{P}\right)+\mathcal{V}=\mathcal{V}_{\mathscr{O}}^{*} \mathscr{H}$ and $\left(\mathcal{V}_{\mathscr{O}}^{*} \mathscr{H} \cap \mathcal{P}\right) \cap \mathcal{V}=\{0\}$. Hence, a state space basis transformation $T$ like the one considered in Lemma 3 can be defined, in particular, by picking $T_{2}$ such that $\operatorname{Im} T_{2}=\mathcal{V}$. Consequently, $A_{12}^{\prime}-B_{1}^{\prime} F_{2}^{\prime}=0$ in (63) and $J_{12}^{\prime}=0$ in (64). Taking into account that such submatrices are zero, one easily gets that $X=0$ solves (66) and (67).

It is worth noting that the reasoning developed in this section only deals with structural notions, thus leaving stability apart from consideration. Indeed, the only requirement on the linear map $F$ considered in Lemma 3 is that of being a friend of $\mathcal{V}_{\mathscr{O}}^{*} \mathscr{H}-$ no stabilization properties are required. Therefore, once the subspace $\mathcal{V}$ satisfying the conditions of Theorem 2 has been determined, if possible, as shown in the proof of sufficiency of Theorem $3, \mathcal{V}$ can be used to synthesize the feedback regulator according to the procedure outlined in the proof of Theorem 2. In particular, this means that a different friend of $\mathcal{V}$ can be used to stabilize the restricted hybrid dynamics $\left.\Sigma_{F}\right|_{\mathcal{P}}$.

\section{A NUMERICAL EXAMPLE}

This section illustrates how to implement the devised procedure by considering the same plant and exogenous system of the numerical example discussed in [22], with the sole exception that the pertubation in [22] is not considered herein. The reason for focusing on this case has been discussed in detail in the Introduction and deals with the fact that this work concerns exact output regulation, while [22] is about almost output regulation. Moreover, the behavior of the closed-loop compensated hybrid system devised herein is compared to that presented in [22], referring to the same initial conditions of [22], but assuming a more stringent sequence of jump times, which, nonetheless, preserves global asymptotic stability of the respective closed-loop hybrid dynamics.

In light of the algorithms and of the constructive proofs of the statements presented so far in this work, the procedure for the synthesis of the hybrid feedback regulator solving the output regulation problem with global asymptotic stability of the compensated hybrid dynamics can be implemented

by resorting to the computational tools for the geometric approach available from [33]. 
Hence, let the extended hybrid system $\Sigma$ be defined by

$$
\begin{aligned}
& A=\left[\begin{array}{cc|cccc}
-1 & -1 & 0 & 0 & 0 & 1 \\
1 & -3 & 0 & 0 & 1 & 0 \\
\hline 0 & 0 & 0 & -3 & 0 & -1 \\
0 & 0 & 3 & 0 & 0 & 0 \\
0 & 0 & 0 & 1 & 0 & 0 \\
0 & 0 & 0 & 0 & 3 & 0
\end{array}\right], \quad B=\left[\begin{array}{c}
0 \\
1 \\
\hline 0 \\
0 \\
0 \\
0
\end{array}\right], \quad J=\left[\begin{array}{cc|cccc}
1.1 & 0 & 0 & 0 & 0 & 0 \\
-1.4 & 1 & 0 & 0 & 0 & 0 \\
\hline 0 & 0 & 1 & 0 & 0 & 0 \\
0 & 0 & 0 & 1 & 0 & 0 \\
0 & 0 & 0 & 0 & 1 & 0 \\
0 & 0 & 0 & 0 & 0 & 1
\end{array}\right], \\
& E=\left[\begin{array}{ll|llll}
1 & 0 & 0 & 0 & -0.02 & -0.03
\end{array}\right], \quad D=0.01, \quad C=\left[\begin{array}{ll|llll}
1 & 0 & 0 & 0 & 0 & 0
\end{array}\right],
\end{aligned}
$$

according to (16)-(17) and the numerical values considered in [22]. Moreover, since this work is focused on dwell time instead of average dwell time, let $\tau$ be the smallest time interval between two consecutive jumps considered in the numerical example of [22]: i.e., let $\tau=2 \mathrm{~s}$.

The solution to the output regulation problem is searched through the sufficient condition expressed by Theorem 2. In order to compute a subspace $\mathcal{V}$ which may satisfy the requisites of Theorem 2, Lemma 3 and Theorem 3 are applied. The subspace $\mathcal{P}$ is defined as $\mathcal{P}=\operatorname{Im} P=\operatorname{Im}\left[I_{2} 0_{2 \times 4}\right]^{\top}$, according to (27). The subspace $\mathcal{V}_{\mathscr{O}}^{*} \mathscr{H}$ coincides with the state space $\mathcal{X}=\mathbb{R}^{6}$, as it turns out by applying Algorithm 1 in light of Proposition 1. Thus, condition (62) is met. A friend of $\mathcal{V}_{\mathscr{O} \mathscr{H}}^{*}$ is given by $F=\left[\begin{array}{llllll}-100 & 0 & 0 & 0 & 2 & 3\end{array}\right]$. The state space basis transformation $T$ considered in Lemma 3 reduces to the identity matrix, owing to $\mathcal{P} \subseteq \mathcal{V}_{\mathscr{O}}^{*} \mathscr{H}$ and $\mathcal{V}_{\mathscr{O} \mathscr{H}}^{*}=\mathcal{X}$. Therefore, the known matrices to consider in (66) and (67) are

$A_{11}^{\prime}+B_{1}^{\prime} F_{1}^{\prime}=\left[\begin{array}{cc}-1 & -1 \\ -99 & -3\end{array}\right], A_{22}^{\prime}=\left[\begin{array}{cccc}0 & -3 & 0 & -1 \\ 3 & 0 & 0 & 0 \\ 0 & 1 & 0 & 0 \\ 0 & 0 & 3 & 0\end{array}\right], A_{12}^{\prime}+B_{1}^{\prime} F_{2}^{\prime}=\left[\begin{array}{cccc}0 & 0 & 0 & 1 \\ 0 & 0 & 3 & 3\end{array}\right]$, $J_{11}^{\prime}=\left[\begin{array}{cc}1.1 & 0 \\ -1.4 & 1\end{array}\right], \quad J_{22}^{\prime}=I_{4}, \quad J_{12}^{\prime}=0_{2 \times 4}$.

As is easy to check, a matrix $X$ that solves (66) and (67) is

$$
X=\left[\begin{array}{llll}
0 & 0 & 0 & 0 \\
0 & 0 & 0 & 1
\end{array}\right]
$$

Hence, a subspace $\mathcal{V}$ satisfying the conditions of Theorem 2 is given by $\mathcal{V}=\operatorname{Im} V=\operatorname{Im}\left[X^{\top} I_{4}\right]^{\top}$ with respect to the original coordinates. Consequently, the design of the feedback regulator can be performed as outlined in the proof of sufficiency of Theorem 2. All the friends of $\mathcal{V}$ can be expressed as $F=\left[\begin{array}{llllll}f_{1} & f_{2} & 0 & 0 & 2 & \left(3-f_{2}\right)\end{array}\right]$, where, according to Lemma 2, $f_{1}$ and $f_{2}$ are free parameters. In particular, the restricted dynamics $\left.\Sigma_{F}\right|_{\mathcal{P}}$ can be rendered globally asymptotically stable over $\mathscr{T}_{2}$ by 
choosing suitable values for $f_{1}$ and $f_{2}$, since the pair $\left(A_{P}, B_{P}\right)$ is controllable - note that, according to (16), $A_{P}$ is the submatrix at the upper left corner of $A$ and $B_{P}$ is the upper submatrix of $B$. Indeed, $f_{1}=0$ and $f_{2}=0$ guarantee global asymptotic stability of the hybrid dynamics $\left.\Sigma_{F}\right|_{\mathcal{P}}$ over $\mathscr{T}_{2}$, by virtue of Lemma 4 in the Appendix. In fact, $A_{P}$ is Hurwitz stable and satisfies the condition $\left\|e^{A_{P} t}\right\| \leq \mu e^{-\lambda t}$ for all $t>0$ with $\mu=1$ and $\lambda=1$. For these values of $\mu$ and $\lambda$, with $\tau=2$ and the given $J_{P}$, it ensues that $\left\|J_{P}\right\| \mu e^{-\lambda \tau}=0.2658<1$. As to global asymptotic stabilization over $\mathscr{T}_{2}$ of the hybrid linear dynamics $\Sigma_{G}$, this can be achieved by a suitable choice of the output injection $G$, according to Proposition 3 in the Appendix, since the pair $(A, C)$ is observable. By applying the procedure described in the constructive proof of Proposition 2 to the dual pair $\left(A^{\top}, C^{\top}\right)$, one gets that a suitable value for the crucial parameter is $\bar{\lambda}=-19$ and, consequently, the stabilizing matrix $G$ is given by

$$
G=\left[\begin{array}{c}
-1.2500 \cdot 10^{2} \\
5.5406 \cdot 10^{4} \\
-3.0609 \cdot 10^{6} \\
-7.8654 \cdot 10^{5} \\
-1.7173 \cdot 10^{5} \\
4.8869 \cdot 10^{4}
\end{array}\right] .
$$

It is just worth mentioning that, under the conditions of Proposition 2 (or, respectively, Proposition 3), global asymptotic stabilization over $\mathscr{T}_{\tau}$ of the considered hybrid linear dynamics can be achieved for any given $\tau$. Hence, the hybrid feedback regulator $\Sigma_{R}$ remains determined in light of (39) and its matrices are

$$
\begin{aligned}
& A_{R}=\left[\begin{array}{cccccc}
-1.2600 \cdot 10^{2} & -1 & 0 & 0 & 0 & 1 \\
5.5407 \cdot 10^{4} & -3 & 0 & 0 & 3 & 3 \\
-3.0609 \cdot 10^{6} & 0 & 0 & -3 & 0 & -1 \\
-7.8654 \cdot 10^{5} & 0 & 3 & 0 & 0 & 0 \\
-1.7173 \cdot 10^{5} & 0 & 0 & 1 & 0 & 0 \\
4.8869 \cdot 10^{4} & 0 & 0 & 0 & 3 & 0
\end{array}\right], \quad B_{R}=\left[\begin{array}{c}
-1.2500 \cdot 10^{2} \\
5.5406 \cdot 10^{4} \\
-3.0609 \cdot 10^{6} \\
-7.8654 \cdot 10^{5} \\
-1.7173 \cdot 10^{5} \\
4.8869 \cdot 10^{4}
\end{array}\right] \text {, } \\
& J_{R}=\left[\begin{array}{cccccc}
1.1 & 0 & 0 & 0 & 0 & 0 \\
-1.4 & 1 & 0 & 0 & 0 & 0 \\
0 & 0 & 1 & 0 & 0 & 0 \\
0 & 0 & 0 & 1 & 0 & 0 \\
0 & 0 & 0 & 0 & 1 & 0 \\
0 & 0 & 0 & 0 & 0 & 1
\end{array}\right] \\
& C_{R}=\left[\begin{array}{llllll}
0 & 0 & 0 & 0 & 2 & 3
\end{array}\right], \quad D_{R}=0 .
\end{aligned}
$$




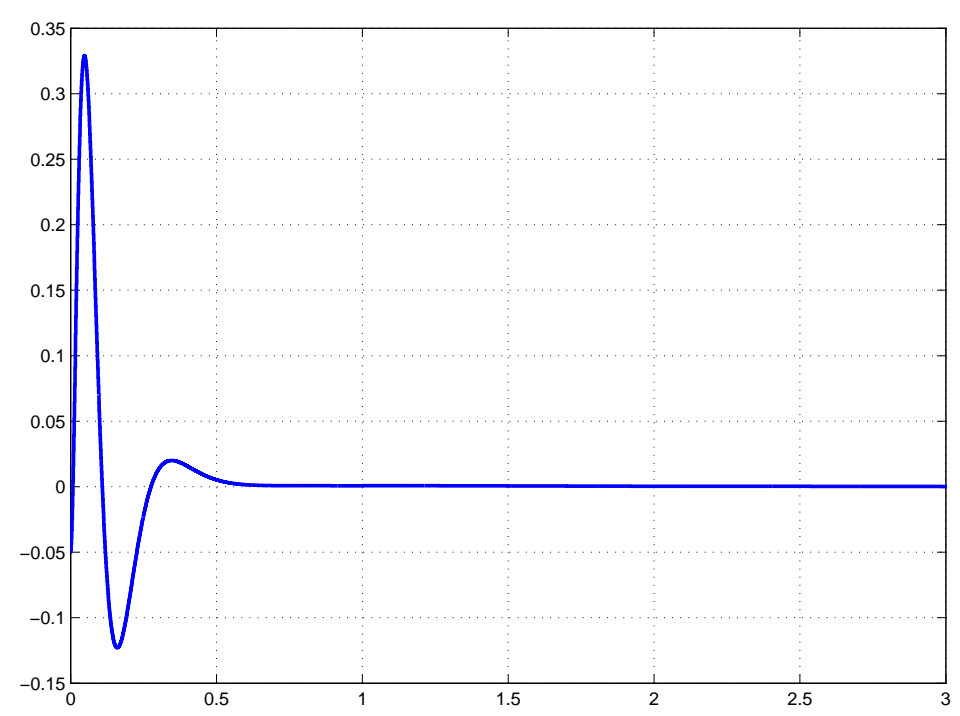

Figure 1. Regulation error - Simulation from time $0 \mathrm{~s}$ to $3 \mathrm{~s}$

The behavior of the closed-loop hybrid system has been checked by running a simulation lasting $10 \mathrm{~s}$, with the same initial conditions considered in [22]: i.e., the initial states of both the plant and the feedback regulator are assumed to be zero, while the initial state of the exogenous system is assumed to be a vector of ones. Instead, the state jumps are assumed to occur every $\tau_{p}=1 \mathrm{~s}$. It is worth noting that, even if the period of such jump time sequence is smaller than the considered minimum dwell time, the closed-loop hybrid dynamics is still globally asymptotically stable, since the eigenvalues of the state transition matrix over one period - i.e., $J_{L} e^{A_{L} \tau_{p}}$, where $A_{L}$ and $J_{L}$ are defined according to (3) and (4) — lie inside the open unit disc of the complex plane. Indeed, this fact is not in contrast with the results discussed in Appendix, since Lemma 4 provides a sufficient (not necessary) condition. Figure 1 shows the plot of the regulation error during the first $3 \mathrm{~s}$ of the simulation. This initial part of the simulation shows that the initial transient becomes negligible within the first second, while the effects on the regulation error of the state jumps occurring every $1 \mathrm{~s}$ are not appreciable in comparison with the initial transient. Figure 2 shows the regulation error between $0.9 \mathrm{~s}$ and $10 \mathrm{~s}$, so that the effects of the state jumps on the regulation error can be made visible on a scale of order $10^{-4}$. For the sake of comparison, Figure 3 shows the regulation error during the entire simulation for both the compensated hybrid system devised herein (solid line) and the compensated hybrid system devised in [22] (dashed line). It is just worth mentioning that also [22] provides sufficient-only conditions for global asymptotic stability of the closed-loop hybrid dynamics and that, in the numerical example, such conditions lead to guarantee global asymptotic stability for an average dwell time of $6.9315 \mathrm{~s}$. However, as it turns out by evaluating the eigenvalues 


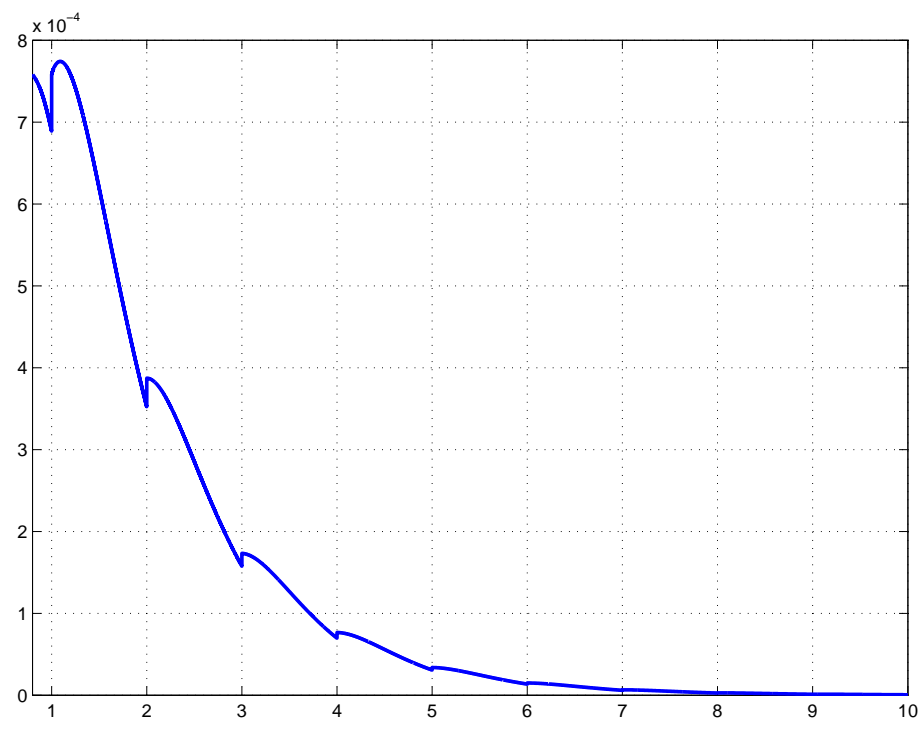

Figure 2. Regulation error - Simulation from time $0.9 \mathrm{~s}$ to $10 \mathrm{~s}$

of the state transition matrix over one period for the closed-loop hybrid dynamics, the latter is globally asymptotically stable also for the considered periodic sequence.

\section{CONCLUSIONS}

This work has presented a solution to the problem of designing a hybrid dynamic feedback regulator that achieves the following objectives: i) forcing the output of a hybrid linear plant to asymptotically converge to the reference generated by a hybrid exogenous system; ii) rejecting the exogenous disturbance; iii) attaining global asymptotic stability of the compensated hybrid dynamics for all the sequences of jump times with a minimum dwell time. A sufficient condition for problem solvability has first been stated implicitly, with reference to the autonomous hybrid system, which includes the plant, the exogenous system, and the regulator. Then, an explicit sufficient condition has been stated with reference to the connection between the hybrid plant and the hybrid exogenous system. The proof of the explicit sufficient condition shows how to synthesize the hybrid feedback regulator, given a subspace that enjoys the properties specified in the statement. Then, a necessary and sufficient condition for the existence of the so-called resolving subspace has been proven. The proof of sufficiency of this latter condition is constructive. Thus, it provides the means to compute the resolving subspace, if it exists. The implementation of the proposed approach has been illustrated by applying it to a numerical example borrowed from the literature. The methodology discussed so far is likely to extend to more complex hybrid systems than those considered herein, such as linear 


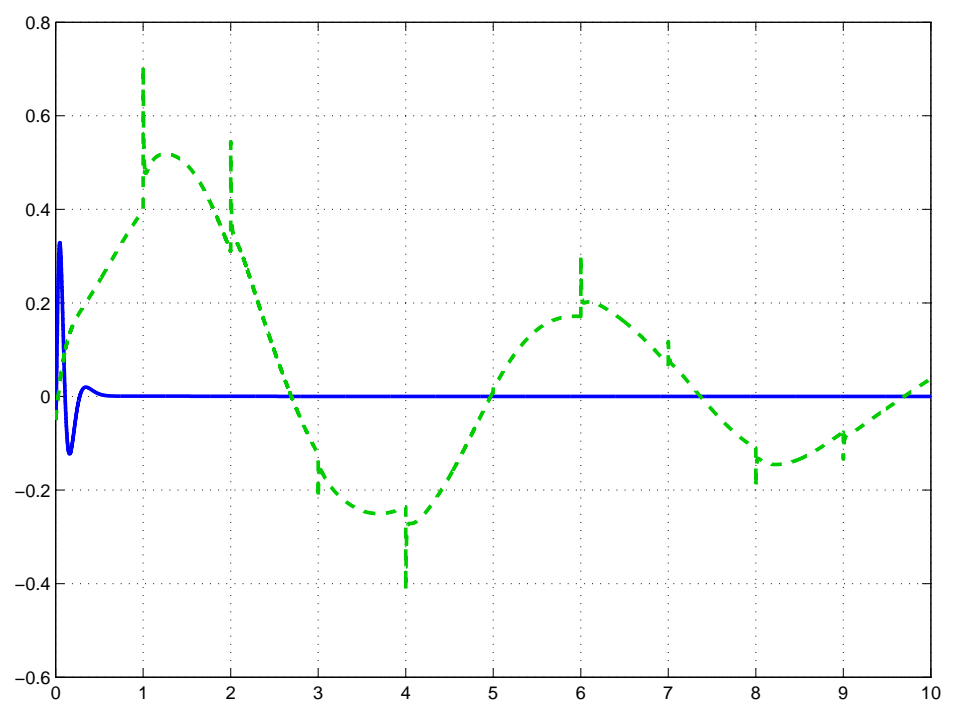

Figure 3. Comparison between the regulation errors - Simulation from time $0 \mathrm{~s}$ to $10 \mathrm{~s}$

parameter varying hybrid systems in particular. The investigation of this topic will be the object of future work.

\section{A. APPENDIX}

This Appendix has a twofold objective. The first aim is to show how the assumption of reachability of the flow dynamics allows global asymptotic stability over $\mathscr{T}_{\tau}$ (with $\tau$ given) of the hybrid linear dynamics to be achieved by means of a static state feedback applied to the flow dynamics only. The second target is to recognize that global asymptotic stability over $\mathscr{T}_{\tau}$ of the hybrid linear dynamics can be achieved by means of an output injection on the flow dynamics, under the assumption of observability of the same flow dynamics. Actually, stabilization of the hybrid linear dynamics can be attained even if the assumption of reachability (or, respectively, observability) of the flow dynamics is replaced by other hypotheses - see, e.g., [4, 15]. However, to the best of the authors' knowledge, none of the methods available from the literature provides a stabilizing static state feedback (or, respectively, output injection) acting on the flow dynamics only. For ease of reference, the next statements, which concern static state feedback, address the hybrid linear plant $\Sigma_{P}$, whose stabilization is required for the design of the hybrid feedback regulator in the proof of Theorem 2.

\section{Lemma 4}

Let the hybrid linear system $\Sigma_{P}$ and the positive real constant $\tau$ be given. Let $A_{P}$ be Hurwitz stable and let $\lambda, \mu \in \mathbb{R}^{+}$be such that $\left\|e^{A_{P} t}\right\| \leq \mu e^{-\lambda t}$ for all $t>0$. Let $J_{P}$ be such that $\left\|J_{P}\right\| \mu e^{-\lambda \tau}<1$. Then, $\Sigma_{P}$ is globally asymptotically stable over $\mathscr{T}_{\tau}$. 
Proof

Let $\mathcal{T}$ be any finite set in $\mathscr{T}_{\tau}$. Then, with $t>t_{f}$, the hybrid linear dynamics of $\Sigma_{P}$ reduces to the flow dynamics, which is (globally) asymptotically stable by assumption. Let $\mathcal{T}$ be any countably infinite set in $\mathscr{T}_{\tau}$. Let $\alpha=\left\|J_{P}\right\| \mu e^{-\lambda \tau}$. First, note that $\left\|J_{P} e^{A_{P} t}\right\| \leq\left\|J_{P}\right\| \mu e^{-\lambda t} \leq\left\|J_{P}\right\| \mu e^{-\lambda \tau}=\alpha<1$, for all $t \geq \tau$. Then, consider the state motion $x_{P}(t)$, with $t \in \mathbb{R}^{+}$, starting from any initial state $x_{P 0}$ in $\mathcal{X}_{P}$. With $t_{k} \in \mathcal{T}$, the state is $x_{P}\left(t_{k}\right)=J_{P} e^{A_{P}\left(t_{k}-t_{k-1}\right)} \ldots J_{P} e^{A_{P}\left(t_{1}-t_{0}\right)} J_{P} e^{A_{P} t_{0}} x_{P 0}$. Therefore, $\left\|x_{P}\left(t_{k}\right)\right\| \leq$ $\left\|J_{P} e^{A_{P}\left(t_{k}-t_{k-1}\right)}\right\| \ldots\left\|J_{P} e^{A_{P}\left(t_{1}-t_{0}\right)}\right\|\left\|J_{P} e^{A_{P} t_{0}}\right\|\left\|x_{P 0}\right\| \leq \alpha^{k+1}\left\|x_{P 0}\right\|$, for all $t_{k} \in \mathcal{T}$, which implies $\left\|x_{P}\left(t_{k}\right)\right\| \rightarrow 0$ as $k \rightarrow \infty$. With $t \in\left(t_{k}, t_{k+1}\right)$, the state is $x_{P}(t)=e^{A_{P}\left(t-t_{k}\right)} x_{P}\left(t_{k}\right)$. Thus, $\left\|x_{P}(t)\right\| \leq\left\|e^{A_{P}\left(t-t_{k}\right)}\right\|\left\|x_{P}\left(t_{k}\right)\right\| \leq \mu\left\|x_{P}\left(t_{k}\right)\right\|$ for all $t \in\left(t_{k}, t_{k+1}\right)$.

\section{Proposition 2}

Let the hybrid linear plant $\Sigma_{P}$ and the positive real constant $\tau$ be given. Let $\left(A_{P}, B_{P}\right)$ be reachable. Then, there exists a linear map $F_{P}: \mathcal{X}_{P} \rightarrow \mathcal{U}$ such that the compensated hybrid linear system $\Sigma_{P F_{P}}$, defined by ${ }^{\dagger}$

$$
\Sigma_{P F_{P}} \equiv\left\{\begin{aligned}
\dot{x}_{P}(t) & =\left(A_{P}+B_{P} F_{P}\right) x_{P}(t), \quad t \in \mathbb{R}^{+} \backslash \mathcal{T} \\
x_{P}\left(t_{k}\right) & =J_{P} x_{P}^{-}\left(t_{k}\right), \quad t_{k} \in \mathcal{T},
\end{aligned}\right.
$$

is globally asymptotically stable over $\mathscr{T}_{\tau}$.

\section{Proof}

First, note that, since $\left(A_{P}, B_{P}\right)$ is reachable, there exists a linear map $F_{P 1}: \mathcal{X}_{P} \rightarrow \mathcal{U}$, such that $A_{P}+B_{P} F_{P 1}$ is cyclic with respect to a column of $B_{P}-$ e.g., the first column, henceforth denoted by $b$. Let $S$ be a similarity transformation such that the pair $\left(A_{P}^{\prime}+B_{P}^{\prime} F_{P 1}^{\prime}, b^{\prime}\right)$, where $A_{P}^{\prime}+B_{P}^{\prime} F_{P 1}^{\prime}=S^{-1}\left(A_{P}+B_{P} F_{P 1}\right) S$ and $b^{\prime}=S^{-1} b$, is in controllable canonical form. Moreover, let $\lambda$ be a positive real parameter and let $F_{P 2}: \mathcal{X}_{P} \rightarrow \mathcal{U}$ be a linear map such that the eigenvalues of $A_{P}+B_{P} F_{P 1}+B F_{P 2}$ are given by the sequence $\lambda_{i}=-\lambda-i+1$, with $i=1,2, \ldots, n_{P}$, and, in the new coordinates, the matrix

$$
A_{P}^{\prime}+B_{P}^{\prime} F_{P 1}^{\prime}+B_{P}^{\prime} F_{P 2}^{\prime}=S^{-1}\left(A_{P}+B_{P} F_{P 1}+B_{P} F_{P 2}\right) S
$$

\footnotetext{
${ }^{\dagger}$ Note that the exogenous input $x_{E}(t)$ and the outputs $y_{R}(t)$ and $y_{M}(t)$, originally present in the definition of $\Sigma_{P}$, are dropped from the definition of $\Sigma_{P F_{P}}$, because they do not play any role in connection with the stabilization by state feedback of the hybrid linear dynamics associated to $A_{P}+B_{P} F_{P}$ and $J_{P}$.
} 
is in companion form - the two requirements are compatible since $A_{P}^{\prime}+B_{P}^{\prime} F_{P 1}^{\prime}$ is cyclic with respect to the first column of $B_{P}^{\prime}$. Let $L_{P}=\operatorname{diag}\left\{\lambda_{1}, \ldots, \lambda_{n_{P}}\right\}$ and let

$$
Q=\left[\begin{array}{cccc}
1 & 1 & \ldots & 1 \\
\lambda_{1} & \lambda_{2} & \ldots & \lambda_{n_{P}} \\
\vdots & \vdots & & \vdots \\
\lambda_{1}^{n_{P}-1} & \lambda_{2}^{n_{P}-1} & \ldots & \lambda_{n_{P}}^{n_{P}-1}
\end{array}\right]
$$

so that

$$
A_{P}^{\prime}+B_{P}^{\prime} F_{P 1}^{\prime}+B_{P}^{\prime} F_{P 2}^{\prime}=Q L_{P} Q^{-1}
$$

Hence,

$$
A_{P}+B_{P} F_{P}=S Q L_{P} Q^{-1} S^{-1}
$$

follows from (69) and (70), with $F_{P}=F_{P 1}+F_{P 2}$. From (71), it follows that

$$
e^{\left(A_{P}+B_{P} F_{P}\right) t}=S Q e^{L_{P} t} Q^{-1} S^{-1}
$$

for all $t>0$. Thus, from (72), it ensues that

$$
\left\|e^{\left(A_{P}+B_{P} F_{P}\right) t}\right\| \leq\|S\|\|Q\|\left\|e^{L_{P} t}\right\|\left\|Q^{-1}\right\|\left\|S^{-1}\right\|
$$

for all $t>0$. Note that, in (73), $\|S\|$ and $\left\|S^{-1}\right\|$ are positive real constants, while $\left\|e^{L_{P} t}\right\|,\|Q\|$, and $\left\|Q^{-1}\right\|$ depend on the parameter $\lambda$. In particular, note that

$$
\left\|e^{L_{P} t}\right\| \leq e^{-\lambda t}
$$

for all $t>0$, since $-\lambda=\lambda_{1}$ is the maximum eigenvalue of the assigned set. Moreover, let

$$
\mu(\lambda)=\|S\|\|Q\|\left\|Q^{-1}\right\|\left\|S^{-1}\right\| .
$$

From (73), (74), and (75), it follows that

$$
\left\|e^{\left(A_{P}+B_{P} F_{P}\right) t}\right\| \leq \mu(\lambda) e^{-\lambda t},
$$

for all $t>0$. For any given $t>0$, the right-hand side argument of (76) can be made arbitrary small by picking a sufficiently large value for the parameter $\lambda$, since, in the worst case, as $\lambda$ increases, $\mu(\lambda)$ increases as a power of $\lambda$, while $e^{-\lambda t}$ decreases exponentially. So, let $\bar{\lambda}$ be such that

$$
\left\|J_{P}\right\| \mu(\bar{\lambda}) e^{-\bar{\lambda} \tau}<1 .
$$

For $\lambda=\bar{\lambda}$, (76) becomes

$$
\left\|e^{\left(A_{P}+B_{P} F_{P}\right) t}\right\| \leq \mu(\bar{\lambda}) e^{-\bar{\lambda} t},
$$

for all $t>0$. Therefore, (77) and (78) completes the proof, by virtue of Lemma 4. 
The following statement concerns global asymptotic stabilization of the hybrid linear dynamics through an output injection applied to the flow dynamics, on the assumption that the flow dynamics is observable. For the sake of immediacy, the statement is given with reference to the hybrid linear system $\Sigma$ where the measured output $e_{M}(t)$ only is taken into consideration, as is done in regard to stabilization by output injection in the proof of Theorem 2 .

\section{Proposition 3}

Let the hybrid linear plant $\Sigma$ and the positive real constant $\tau$ be given. Let $(A, C)$ be observable. Then, there exists a linear map $G: \mathcal{Y} \rightarrow \mathcal{X}$ such that the compensated hybrid linear system $\Sigma_{G}$ is globally asymptotically stable over $\mathscr{T}_{\tau}$.

Proof

It can be derived from Proposition 2 by duality arguments.

\section{REFERENCES}

1. Li Z, Soh Y, Wen C. Switched and Impulsive Systems: Analysis, Design and Applications, Lecture Notes in Control and Information Sciences, vol. 313. Springer-Verlag: Berlin Heidelberg, 2005.

2. Haddad WM, Chellaboina VS, Nersesov SG. Impulsive and Hybrid Dynamical Systems: Stability, Dissipativity, and Control, Princeton Series in Applied Mathematics, vol. 6. Princeton University Press: Princeton, NJ, 2006.

3. Goebel R, Sanfelice RG, Teel AR. Hybrid Dynamical Systems: Modeling, Stability, and Robustness. Princeton University Press: Princeton, NJ, 2012.

4. Medina EA, Lawrence DA. State feedback stabilization of linear impulsive systems. Automatica 2009; 45(6):14761480 .

5. Medina EA, Lawrence DA. Output feedback stabilization for linear impulsive systems. 2010 American Control Conference, Baltimore, MD, 2010; 4211-4216.

6. Lawrence DA. Lie-algebraic conditions for stability of linear impulsive systems. 2013 American Control Conference, Washington, DC, 2013; 3248-3253.

7. Medina EA, Lawrence DA. State estimation for linear impulsive systems. 2009 American Control Conference, St. Louis, MO, 2009; 1183-1188.

8. Kouhi Y, Bajcinca N, Sanfelice RG. Suboptimality bounds for linear quadratic problems in hybrid linear systems. 2013 European Control Conference, Zurich, Switzerland, 2013; 2663-2668.

9. Carnevale D, Galeani S, Sassano M. A linear quadratic approach to linear time invariant stabilization for a class of hybrid systems. 22nd Mediterranean Conference on Control and Automation, Palermo, Italy, 2014; 545-550.

10. Conte G, Perdon AM, Zattoni E. The disturbance decoupling problem for jumping hybrid systems. 54th IEEE Conference on Decision and Control, Osaka, Japan, 2015; 1589-1594.

11. Perdon AM, Zattoni E, Conte G. Disturbance decoupling with stability for linear impulsive systems. 6th IFAC Symposium on System Structure and Control, Istanbul, Turkey, 2016; 1-6.

12. Perdon AM, Zattoni E, Conte G. Disturbance decoupling in hybrid linear systems with state jumps 2016. Submitted.

13. Medina EA, Lawrence DA. Controlled and conditioned invariants for linear impulsive systems. 45th IEEE Conference on Decision and Control, San Diego, CA, 2006; 2753-2758.

14. Carnevale D, Galeani S, Menini L. Output regulation for a class of linear hybrid systems. Part 1: Trajectory generation. 51st IEEE Conference on Decision and Control, Maui, HI, 2012; 6151-6156. 
15. Carnevale D, Galeani S, Menini L. Output regulation for a class of linear hybrid systems. Part 2: Stabilization. 51st IEEE Conference on Decision and Control, Maui, HI, 2012; 6157-6162.

16. Carnevale D, Galeani S, Sassano M. Necessary and sufficient conditions for output regulation in a class of hybrid linear systems. 52nd IEEE Conference on Decision and Control, Florence, Italy, 2013; 2659-2664.

17. Carnevale D, Galeani S, Menini L, Sassano M. Hybrid output regulation for linear systems with periodic jumps: Solvability conditions, structural implications and semi-classical solutions. IEEE Transactions on Automatic Control 2016; 61(9):2416-2431.

18. Zattoni E, Perdon AM, Conte G. Output regulation by error dynamic feedback in linear time-invariant hybrid dynamical systems. European Control Conference 2015, Linz, Austria, 2015; 172-177.

19. Zattoni E, Perdon AM, Conte G. Output regulation by error dynamic feedback in hybrid systems with periodic state jumps. Automatica 2017; 53(8), doi:10.1016/j.automatica.2017.03.037. In press.

20. Carnevale D, Galeani S, Menini L, Sassano M. Output regulation of hybrid linear systems with unpredictable jumps. 19th IFAC World Congress, Cape Town, South Africa, 2014; 1531-1536.

21. Carnevale D, Galeani S, Sassano M. Francis equations vs invariant subspace algorithm for hybrid output regulation. 53rd IEEE Conference on Decision and Control, Los Angeles, CA, 2014; 4697-4702.

22. Yuan C, Wu F. Hybrid almost output regulation of linear impulsive systems with average dwell time. Nonlinear Analysis: Hybrid Systems 2016; 20:82-94.

23. Amato F, De Tommasi G, Pironti A. Input-output finite-time stabilization of impulsive linear systems: Necessary and sufficient conditions. Nonlinear Analysis: Hybrid Systems 2016; 19:93-106.

24. Amato F, Carannante G, De Tommasi G, Pironti A. Finite-time control of switching linear systems: The uncertain resetting time case. International Journal of Robust and Nonlinear Control 2015; 25:2547-2560.

25. Zattoni E, Perdon AM, Conte G. The output regulation problem with stability for linear switching systems: A geometric approach. Automatica 2013; 49(10):2953-2962.

26. Perdon AM, Conte G, Zattoni E. Necessary and sufficient conditions for asymptotic model matching of switching linear systems. Automatica 2016; 64(2):294-304.

27. Zattoni E, Perdon AM, Conte G. Disturbance decoupling with closed-loop modes stability in switched linear systems. IEEE Transactions on Automatic Control 2016; 61(10):3115-3121.

28. Perdon AM, Zattoni E, Conte G. Model matching with strong stability in switched linear systems. Systems \& Control Letters 2016; 97:98-107.

29. Francis B, Sebakhy OA, Wonham WM. Synthesis of multivariable regulators: The internal model principle. Applied Mathematics and Optimization 1974; 1(1):64-86.

30. Marro G. Multivariable regulation in geometric terms: Old and new results. Colloquium on Automatic Control, Lecture Notes in Control and Information Sciences, vol. 215. Springer-Verlag: London, 1996; 77-138.

31. Lawrence DA. Controlled invariant subspaces for linear impulsive systems. 2014 American Control Conference, Portland, OR, 2014; 2336-2341.

32. Lawrence DA. Conditioned invariant subspaces for linear impulsive systems. 2015 American Control Conference, Chicago, IL, 2015; 4850-4855.

33. Basile G, Marro G. Controlled and Conditioned Invariants in Linear System Theory. Prentice Hall: Englewood Cliffs, NJ, 1992. 